\title{
Wittgenstein's Picture Theory in the Quantum Mechanical Worldview
}

\author{
Shiro Ishikawa \\ Department of Mathematics, Faculty of Science and Technology, Keio University, Yokohama, Japan \\ Email: ishikawa@math.keio.ac.jp
}

How to cite this paper: Ishikawa, S. (2020) Wittgenstein's Picture Theory in the Quantum Mechanical Worldview. Journal of Quantum Information Science, 10, 104-125. https://doi.org/10.4236/jqis.2020.104007

Received: November 29, 2020

Accepted: December 27, 2020

Published: December 30, 2020

Copyright $\odot 2020$ by author(s) and Scientific Research Publishing Inc. This work is licensed under the Creative Commons Attribution International License (CC BY 4.0).

http://creativecommons.org/licenses/by/4.0/ (c) (i) Open Access

\begin{abstract}
Recently we proposed the linguistic Copenhagen interpretation (or, quantum language), which has a great power to describe both classical and quantum systems. Thus we think that quantum language can be viewed as the language of science. Therefore, it makes sense to study, from the quantum linguistic point of view, Wittgenstein's picture theory, since he must have wanted to create a language of science. In this paper, we show that the proposition that Wittgenstein studied in his book "Tractatus Logico-Philosophicus" can be regarded as a binary projective measurement in classical quantum language. And thus, we conclude that Wittgenstein's language (i.e., the language that he supposed in his book) is realized by classical quantum language. Hence, now we can fully understand Wittgenstein's picture theory since the reason his book is incomprehensible is that he did not define his language.
\end{abstract}

\section{Keywords}

Wittgenstein's Picture Theory, Quantum Language, Linguistic Copenhagen Interpretation

\section{Introduction}

Wittgenstein's book "Tractatus Logico-Philosophicus" (in short, “TLP”; ref. [1]) is surely one of the most important philosophical books of the $20^{\text {th }}$ century. However, there are various opinions about his picture theory (which is the main theory in TLP). For example, some people have even declared his picture theory to be nonsensical (cf. refs. [2] [3]). Therefore, I don't think the evaluation of his theory is yet settled from the scientific point of view. He said, in the (6.53)-th sentence of TLP, that

- (6.53): The correct method in philosophy is to say nothing but what can be said (i.e., propositions of the natural sciences). 
Therefore, he must have been thinking about the language of science to answer "what is a scientific proposition?"

For almost 30+ years, I've been studying quantum language (abbreviated and cited as QL) (or, "measurement theory", "the linguistic Copenhagen interpretation of quantum mechanics", "the quantum mechanical worldview"), which was proposed by myself ( $c f$. refs. [4] [5] [6] [7] [8]). As seen in ref. [9] (in which most of my results concerning QL are written), roughly speaking, QL has three aspects such as the linguistic turn of quantum mechanics (cf. refs. [10] [11] [12]), the scientific turn of Descartes=Kant philosophy (cf. refs. [13] [14] [15]) and the dualistic turn of statistics (cf. refs. [16] [17] [18] [19] [20]). And thus, it has a great power to solve many philosophical unsolved problems (e.g., Zeno's paradox, Hume's problem of induction, Hempel's raven problem, etc. $c f$. Chap. 12 in ref. [21]). Thus, I think that QL is a language of science.

The purpose of this paper is to propose my understanding of Wittgenstein's picture theory in the framework of QL. This is reasonable since both Wittgenstein's picture theory and QL are closely related to propositions of science. In Section 2, I review measurement theory, which is composed from Axiom 1 (Measurement), Axiom 2 (Causality) and the linguistic Copenhagen interpretation. In Section 3, I discuss "Why does logic arise in classical system?", and assert that a class of classical binary projective measurements has a logical structure. In Section 4, I study Wittgenstein's picture theory, which is constructed on inspiration from the results obtained in Section 3. In Section 5, I note that syllogism does not always holds in quantum systems. After all, I conclude that the proposition Wittgenstein studied in his TLP can be regarded as a binary projective measurement in classical quantum language. Therefore, we think that Wittgenstein's language (i.e., the language that he supposed in TLP) is realized by classical QL.

\section{Review: $Q$ uantum Language $(=\mathrm{QL}=$ Measurement $T$ heory $)$}

In this section, we shall review quantum language (i.e., the linguistic Copenhagen interpretation of quantum mechanics, or measurement theory), which has the following form:

$$
\begin{aligned}
& \frac{\text { Quantumlanguage }}{\text { (=measurement theory) }} \\
& =\frac{\text { measurement }}{\text { (Axiom 1) }}+\frac{\text { Causality }}{\text { (Axiom 2) }}+\frac{\text { linguistic(Copenhagen) interpretation }}{\text { (how to use Axioms 1 and 2) }}
\end{aligned}
$$

My all results concerning QL are summarized in Research report (Keio University): [9] [21]. Also, the sections 4.3 and 11.6 in ref. [21] should be regarded as the preprint of this paper.

\subsection{Mathematical Preparations}

Consider an operator algebra $B(H)$ (i.e., an operator algebra composed of all bounded linear operators on a Hilbert space $H$ with the norm

$\|F\|_{B(H)}=\sup _{\|u\|_{H}=1}\|F u\|_{H}$, [22] [23] [24]), and consider the pair $[\mathcal{A}, \mathcal{N}]_{B(H)}$ (or, 
the triplet $[\mathcal{A} \subseteq \mathcal{N} \subseteq B(H)])$, called a basic structure. Here, $\mathcal{A}(\subseteq B(H))$ is a $C$-algebra, and $\mathcal{N}(\mathcal{A} \subseteq \mathcal{N} \subseteq B(H))$ is a particular $C$-algebra (called a $W^{*}$-algebra) such that $\mathcal{N}$ is the weak closure of $\mathcal{A}$ in $B(H)$.

QL (= quantum language $=$ measurement theory) is classified as follows.

(A) $\mathrm{QL}= \begin{cases}\left(\mathrm{A}_{1}\right): \text { quantum QL } & (\text { when } \mathcal{A}=\mathcal{C}(H)) \\ \left(\mathrm{A}_{2}\right): \text { classical QL } & \left(\text { when } \mathcal{A}=C_{0}(\Omega)\right)\end{cases}$

That is, when $\mathcal{A}=\mathcal{C}(H)$, the $C$-algebra composed of all compact operators on a Hilbert space $H$, the quantum QL $\left(\mathrm{A}_{1}\right)$ is also called quantum measurement theory (or, quantum system theory), which can be regarded as the linguistic aspect of quantum mechanics. Also, when $\mathcal{A}$ is commutative (that is, when $\mathcal{A}$ is characterized by $C_{0}(\Omega)$, the $C$-algebra composed of all continuous complex-valued functions vanishing at infinity on a locally compact Hausdorff space $\Omega$ (cf. refs. [23] [24])), the classical QL $\left(\mathrm{A}_{2}\right)$ is also called classical measurement theory.

Also, note that, when $\mathcal{A}=\mathcal{C}(H)$, i.e., quantum cases,

(i) $\mathcal{A}^{*}=\operatorname{Tr}(H) \quad$ (=trace class), $\mathcal{N}=B(H), \quad \mathcal{N}_{*}=\operatorname{Tr}(H)$ (i.e., pre-dual space), thus, $\operatorname{Tr}_{\operatorname{Tr}(H)}(\rho, T)_{B(H)}=\operatorname{Tr}_{H}(\rho T) \quad(\rho \in \operatorname{Tr}(H), T \in B(H))$.

Also, when $\mathcal{A}=C_{0}(\Omega)$, i.e., classical cases,

(ii) $\mathcal{A}^{*}=\mathcal{M}(\Omega)$ (="the space of all signed measures on $\Omega$ )", $\mathcal{N}=L^{\infty}(\Omega, v)\left(\subseteq B\left(L^{2}(\Omega, v)\right)\right), \quad \mathcal{N}_{*}=L^{1}(\Omega, v)$, where $v$ is some measure on $\Omega$, thus, $\quad L^{1}(\Omega, v)(\rho, T)_{L^{\infty}(\Omega, v)}=\int_{\Omega} \rho(\omega) T(\omega) v(\mathrm{~d} \omega)$ $\left(\rho \in L^{1}(\Omega, v), T \in L^{\infty}(\Omega, v)\right)$ (cf. ref. [23]).

Let $\mathcal{A}(\subseteq \mathcal{N} \subseteq B(H))$ be a $C$-algebra, and let $\mathcal{A}^{*}$ be the dual Banach space of $\mathcal{A}$. That is, $\mathcal{A}^{*}=\{\rho \mid \rho$ is a continuous linear functional on $\mathcal{A}\}$, and the norm $\|\rho\|_{\mathcal{A}^{*}}$ is defined by $\sup \left\{|\rho(F)| \mid F \in \mathcal{A}\right.$ such that $\left.\|F\|_{\mathcal{A}}\left(=\|F\|_{B(H)}\right) \leq 1\right\}$. Define the mixed state $\rho\left(\in \mathcal{A}^{*}\right)$ such that $\|\rho\|_{\mathcal{A}^{*}}=1$ and $\rho(F) \geq 0$ for all $F \in \mathcal{A}$ such that $F \geq 0$. And define the mixed state space $\mathfrak{S}^{m}\left(\mathcal{A}^{*}\right)$ such that

$$
\mathfrak{S}^{m}\left(\mathcal{A}^{*}\right)=\left\{\rho \in \mathcal{A}^{*} \mid \rho \text { is a mixed state }\right\} .
$$

A mixed state $\rho\left(\in \mathfrak{S}^{m}\left(\mathcal{A}^{*}\right)\right)$ is called a pure state if it satisfies that $\rho=\theta \rho_{1}+(1-\theta) \rho_{2} \quad$ for some $\rho_{1}, \rho_{2} \in \mathfrak{S}^{m}\left(\mathcal{A}^{*}\right)$ and $0<\theta<1 \quad$ implies $\rho=\rho_{1}=\rho_{2}$. Put

$$
\mathfrak{S}^{p}\left(\mathcal{A}^{*}\right)=\left\{\rho \in \mathfrak{S}^{m}\left(\mathcal{A}^{*}\right) \mid \rho \text { is a pure state }\right\},
$$

which is called a state space. It is well known (cf. ref. [23]) that $\mathfrak{S}^{p}\left(\mathcal{C}(H)^{*}\right)=\left\{|u\rangle\langle u|(\right.$ i.e., the Dirac notation $\left.) \mid\|u\|_{H}=1\right\}$, and $\mathfrak{S}^{p}\left(C_{0}(\Omega)^{*}\right)=\left\{\delta_{\omega_{0}} \mid \delta_{\omega_{0}}\right.$ is a point measure at $\left.\omega_{0} \in \Omega\right\}$, where $\int_{\Omega} f(\omega) \delta_{\omega_{0}}(\mathrm{~d} \omega)=f\left(\omega_{0}\right) \quad\left(\forall f \in C_{0}(\Omega)\right)$. The latter implies that $\mathfrak{S}^{p}\left(C_{0}(\Omega)^{*}\right)$ can be also identified with $\Omega$ (called a spectrum space or simply spectrum) such as

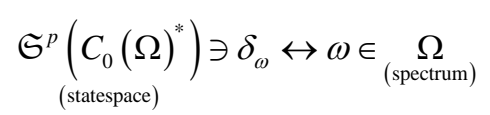


Thus, $\omega$ and $\Omega$ is also called a state and state space respectively.

For instance, in the above (ii) we must clarify the meaning of the value" of $f\left(\omega_{0}\right)$ for $f \in L^{\infty}(\Omega, v)$ and $\omega_{0} \in \Omega$. An element $f\left(\in L^{\infty}(\Omega, v)\right)$ is said to be essentially continuous at $\omega_{0}(\in \Omega)$, if there uniquely exists a complex number $\alpha$ such that

- if $\rho\left(\in L^{1}(\Omega, v),\|\rho\|_{L^{1}(\Omega, v)}=1, \rho \geq 0\right)$ converges to $\delta_{\omega_{0}}(\in \mathcal{M}(\Omega))$ in the sense of weak topology of $\mathcal{M}(\Omega)$, that is,

$$
\rho(G) \rightarrow G\left(\omega_{0}\right)\left(\forall G \in C_{0}(\Omega)\left(\subseteq L^{\infty}(\Omega, v)\right)\right),
$$

then $\rho(f)\left(=\int_{\Omega} f(\omega) \rho(\omega) v(\mathrm{~d} \omega)\right)$ converges to $\alpha$.

And the value of $f\left(\omega_{0}\right)$ is defined by the $\alpha$.

Definition 1. [Observable] According to the noted idea (cf. ref. [25] [26]), an observable $\mathrm{O}:=(X, \mathcal{F}, F)$ in $\mathcal{N}$ is defined as follows:

(i) [ $\sigma$-field $] X$ is a set, $\mathcal{F} \quad\left(\subseteq 2^{X}\right.$, the power set of $\left.X\right)$ is a $\sigma$-field of $X$, that is, “ $\Xi_{1}, \Xi_{2}, \cdots \in \mathcal{F} \Rightarrow \bigcup_{n=1}^{\infty} \Xi_{n} \in \mathcal{F}$ ”, “ $\Xi \in \mathcal{F} \Rightarrow X \backslash \Xi \in \mathcal{F}$ ”.

(ii) [Countable additivity] $F$ is a mapping from $\mathcal{F}$ to $\mathcal{N}$ satisfying: (a): for every $\Xi \in \mathcal{F}, F(\Xi)$ is a non-negative element in $\mathcal{N}$ such that $0 \leq F(\Xi) \leq I,(\mathrm{~b})$ : $F(\varnothing)=0$ and $F(X)=I$, where 0 and $I$ is the 0 -element and the identity in $\mathcal{N}$ respectively. (c): for any countable decomposition $\left\{\Xi_{1}, \Xi_{2}, \cdots, \Xi_{n}, \cdots\right\}$ of $\Xi$ (i.e., $\left.\Xi, \Xi_{n} \in \mathcal{F}(n=1,2,3, \cdots), \quad \bigcup_{n=1}^{\infty} \Xi_{n}=\Xi, \quad \Xi_{i} \cap \Xi_{j}=\varnothing \quad(i \neq j)\right)$, it holds that $F(\Xi)=\sum_{n=1}^{\infty} F\left(\Xi_{n}\right)$ in the sense of weak topology in $\mathcal{N}$.

Let $(Y, \mathcal{G})$ be a measurable space, and let $\Theta: X \rightarrow Y$ be a measurable map. Then, $\Theta(\mathrm{O}):=\left(Y, \mathcal{G}, F\left(\Theta^{-1}(\cdot)\right)\right)$ in $\mathcal{N}$ is also an observable in $\mathcal{N}$ (which is called an image observable). If $F(\Xi)=F(\Xi)^{2} \quad(\forall \Xi \in \mathcal{F})$, then $O:=(X, \mathcal{F}, F)$ in $\mathcal{N}$ is a projective observable $\mathrm{O}:=(X, \mathcal{F}, F)$ in $\mathcal{N}$ is also called an $X$-valued observable. I will devote myself to binary valued (i.e., $\{1,0\}$-valued) projective observables in most of the cases in this paper.

\subsection{Axiom 1 [Measurement] and Axiom 2 [Causality]}

Measurement theory (A) is composed of two axioms (i.e., Axioms 1 and 2) as follows. With any system $S$, a basic structure $[\mathcal{A}, \mathcal{N}]_{B(H)}$ can be associated in which the measurement theory (A) of that system can be formulated. A state of the system $S$ is represented by an element $\rho\left(\in \mathfrak{S}^{p}\left(\mathcal{A}^{*}\right)\right)$ and an observable is represented by an observable $\mathrm{O}:=(X, \mathcal{F}, F)$ in $\mathcal{N}$. Also, the measurement of the observable $\mathrm{O}$ for the system $S$ with the state $\rho$ is denoted by $\mathrm{M}\left(\mathrm{O}, S_{[\rho]}\right)$ (or more precisely, $\mathrm{M}_{\mathcal{N}}\left(\mathrm{O}, S_{[\rho]}\right), \mathrm{M}_{\mathcal{N}}\left(\mathrm{O}:=(X, \mathcal{F}, F), S_{[\rho]}\right)$ ). An observer can obtain a measured value $x(\in X)$ by the measurement $\mathrm{M}\left(\mathrm{O}, S_{[\rho]}\right)$.

The Axiom 1 presented below is a kind of mathematical generalization of Born's probabilistic interpretation of quantum mechanics. And thus, it is a statement without reality.

Now we can present Axiom 1 in the $W^{*}$-algebraic formulation as follows.

Axiom 1 [Measurement]. Consider a basic structure $[\mathcal{A} \subseteq \mathcal{N} \subseteq B(H)]$. The probability that a measured value $x(\in X)$ obtained by the measurement 
$\mathrm{M}_{\mathcal{N}}\left(\mathrm{O}:=(X, \mathcal{F}, F), S_{[\rho]}\right)$ belongs to a set $\Xi(\in \mathcal{F})$ is given by $\rho(F(\Xi))$ if $F(\Xi)$ is essentially continuous at $\rho\left(\in \mathfrak{S}^{p}\left(\mathcal{A}^{*}\right)\right)$.

Remark 2. Recall that a statement whose truth or falsity is determined is called a proposition. Also, Axiom 1 says that a measured value is determined by a measurement $\mathrm{M}_{\mathcal{N}}\left(\mathrm{O}:=(X, \mathcal{F}, F), S_{[\rho]}\right)$. Therefore, there is a point in thinking that "measured value" is a generalization of "truth value". That is, we can regard "measurement" as a kind of generalizations of "proposition". This idea is essential to Sec. 3.

Next, we explain Axiom 2 (which is not used in this paper). Let $\left[\mathcal{A}_{1}, \mathcal{N}_{1}\right]_{B\left(H_{1}\right)}$ and $\left[\mathcal{A}_{2}, \mathcal{N}_{2}\right]_{B\left(H_{2}\right)}$ be basic structures. A continuous linear operator $\Phi_{1,2}: \mathcal{N}_{2}$ (with weak topology) $\rightarrow \mathcal{N}_{1}$ (with weak ${ }^{*}$ topology) is called a Markov operator, if it satisfies that (i): $\Phi_{1,2}\left(F_{2}\right) \geq 0$ for any non-negative element $F_{2}$ in $\mathcal{N}_{2}$, (ii): $\Phi_{1,2}\left(I_{2}\right)=I_{1}$, where $I_{k}$ is the identity in $\mathcal{N}_{k},(k=1,2)$. In addition to the above (i) and (ii), we assume that $\Phi_{1,2}\left(\mathcal{A}_{2}\right) \subseteq \mathcal{A}_{1}$ and $\sup \left\{\left\|\Phi_{1,2}\left(F_{2}\right)\right\|_{\mathcal{A}_{1}} \mid F_{2} \in \mathcal{A}_{2}\right.$ such that $\left.\left\|F_{2}\right\|_{\mathcal{A}_{2}} \leq 1\right\}=1$.

It is clear that the dual operator $\Phi_{1,2}^{*}: \mathcal{A}_{1}^{*} \rightarrow \mathcal{A}_{2}^{*}$ satisfies that $\Phi_{1,2}^{*}\left(\mathfrak{S}^{m}\left(\mathcal{A}_{1}^{*}\right)\right) \subseteq \mathfrak{S}^{m}\left(\mathcal{A}_{2}^{*}\right)$. If it holds that $\Phi_{1,2}^{*}\left(\mathfrak{S}^{p}\left(\mathcal{A}_{1}^{*}\right)\right) \subseteq \mathfrak{S}^{p}\left(\mathcal{A}_{2}^{*}\right)$, the $\Phi_{1,2}$ is said to be deterministic. If it is not deterministic, it is said to be non-deterministic. Also note that, for any observable $\mathrm{O}_{2}:=\left(X, \mathcal{F}, F_{2}\right)$ in $\mathcal{N}_{2}$, the $\left(X, \mathcal{F}, \Phi_{1,2} F_{2}\right)$ is an observable in $\mathcal{N}_{1}$.

Definition 3. [Sequential causal operator; Heisenberg picture of causality] Let $(T, \leq)$ be a tree like semi-ordered set such that $t_{1} \leq t_{3}$ and $t_{2} \leq t_{3}$ implies $t_{1} \leq t_{2}$ or $t_{2} \leq t_{1}$. The family $\left\{\Phi_{t_{1}, t_{2}}: \mathcal{N}_{t_{2}} \rightarrow \mathcal{N}_{t_{1}}\right\}_{\left(t_{1}, t_{2}\right) \in T_{\underline{I}}^{2}}$ is called a sequential causal operator, if it satisfies that

(i) For each $t(\in T)$, a basic structure $\left[\mathcal{A}_{t} \subseteq \mathcal{N}_{t} \subseteq B\left(H_{t}\right)\right]$ is determined.

(ii) For each $\left(t_{1}, t_{2}\right) \in T_{\leqq}^{2}$, a causal operator $\Phi_{t_{1}, t_{2}}: \mathcal{N}_{t_{2}} \rightarrow \mathcal{N}_{t_{1}}$ is defined such as $\Phi_{t_{1}, t_{2}} \Phi_{t_{2}, t_{3}}=\Phi_{t_{1}, t_{3}}\left(\forall\left(t_{1}, t_{2}\right), \forall\left(t_{2}, t_{3}\right) \in T_{\leqq}^{2}\right)$. Here, $\Phi_{t, t}: \mathcal{N}_{t} \rightarrow \mathcal{N}_{t}$ is the identity operator.

Now we can propose Axiom 2 (i.e., causality). (For details, see ref [9].)

Axiom 2 [Causality]; For each $t(\in T=$ "tree like semi-ordered set"), consider the basic structure:

$$
\left[\mathcal{A}_{t} \subseteq \mathcal{N}_{t} \subseteq B\left(H_{t}\right)\right]
$$

Then, the chain of causalities is represented by a sequential causal operator $\left\{\Phi_{t_{1}, t_{2}}: \mathcal{N}_{t_{2}} \rightarrow \mathcal{N}_{t_{1}}\right\}_{\left(t_{1}, t_{2}\right) \in \underline{\underline{\underline{T}}}}$.

\subsection{The Linguistic Copenhagen Interpretation (=The Manual to Use Axioms 1 and 2)}

Since so-called Copenhagen interpretation is not firm (cf. ref. [27]), we propose the linguistic Copenhagen interpretation in what follows. In the above, Axioms 1 and 2 are kinds of spells, (i.e., incantation, magic words, metaphysical statements), and thus, it is nonsense to verify them experimentally. Therefore, what we should do is not to understand" but to use". After learning Axioms 1 and 2 by rote, we have to improve how to use them through trial and error. 
The most important statement in the linguistic Copenhagen interpretation is as follows.

(B) Only one measurement is permitted. Thus, Axiom 1 can be used only once. And therefore, the state after a measurement is meaningless since it cannot be measured any longer. That is, the state is only one.

Referring ref. [9], we have the following definition:

Definition 4. [(i; a)] Simultaneous observable]: Let $\mathrm{O}_{i}:=\left(X_{i}, \mathcal{F}_{i}, F_{i}\right)$ $(i=1,2, \cdots, N)$ be commutative projective observables in $\mathcal{N}$. Let $\left(\times_{i=1}^{N} X_{i}, \bigotimes_{i=1}^{N} \mathcal{F}_{i}\right)$ is a product measurable space of $\left\{\left(X_{i}, \mathcal{F}_{i}\right)\right\}_{i=1}^{N}$. Then, there uniquely exists a projective observable $\times_{i=1}^{N} \mathrm{O}_{i}=\left(\times_{i=1}^{N} X_{i}, \bigotimes_{i=1}^{N} \mathcal{F}_{i}, \times_{i=1}^{N} F_{i}\right)$ such that

$$
\begin{aligned}
& {\left[\begin{array}{c}
N \\
\times \\
i=1
\end{array} F_{i}\right]\left(X_{1} \times X_{2} \times \cdots \times X_{j-1} \times \Xi_{j} \times X_{j+1} \times \cdots \times X_{N}\right)} \\
& =F_{j}\left(\Xi_{j}\right) \quad\left(\forall \Xi_{j} \in \mathcal{F}_{i}, j=1,2, \cdots, N\right)
\end{aligned}
$$

This $\times_{i=1}^{N} \mathrm{O}_{i}$ is called a simultaneous observable (or, product observable) of $\left\{\mathrm{O}_{i} \mid i=1,2, \cdots, N\right\}$. Note that the existence and uniqueness is guaranteed (cf., ref. [9]).

[(i; b)] Simultaneous measurement]: A measurement $\mathrm{M}_{\mathcal{N}}\left(\times_{i=1}^{N} \mathrm{O}_{i}=\left(\times_{i=1}^{N} X_{i}, \bigotimes_{i=1}^{N} \mathcal{F}_{i}, \times_{i=1}^{N} F_{i}\right), S_{[\rho]}\right)$ is called a simultaneous measurement concerning commutative $\mathrm{O}_{i}(i=1,2, \cdots, N)$ in $\mathcal{N}$.

[(ii; a)] Parallel observable]: Let $\mathrm{O}_{i}:=\left(X_{i}, \mathcal{F}_{i}, F_{i}\right)$ be a projective observable in $\mathcal{N}_{i}(i=1,2, \cdots, N)$. Let $\left(\times_{i=1}^{N} X_{i}, \bigotimes_{i=1}^{N} \mathcal{F}_{i}\right)$ is a product measurable space of $\left\{\left(X_{i}, \mathcal{F}_{i}\right)\right\}_{i=1}^{N}$. Then, there uniquely exists an observable $\otimes_{i=1}^{N} \mathrm{O}_{i}=\left(\times_{i=1}^{N} X_{i}, \bigotimes_{i=1}^{N} \mathcal{F}_{i}, \bigotimes_{i=1}^{N} F_{i}\right)$ in a tensor algebra $\otimes_{i=1}^{N} \mathcal{N}_{i}$ such that

$$
\begin{aligned}
& {\left[\otimes_{i=1}^{N} F_{i}\right]\left(X_{1} \times X_{2} \times \cdots \times X_{j-1} \times \Xi_{j} \times X_{j+1} \times \cdots \times X_{N}\right)} \\
& =I_{1} \otimes I_{2} \otimes \cdots \otimes I_{j-1} \otimes F_{i}\left(\Xi_{j}\right) \otimes I_{j+1} \otimes \cdots \otimes I_{N}\left(\forall \Xi_{j} \in \mathcal{F}_{j}, j=1,2, \cdots, N\right)
\end{aligned}
$$

This $\otimes_{i=1}^{N} \mathrm{O}_{i}$ is called a parallel observable (or, tensor observable) of $\left\{\mathrm{O}_{i} \mid i=1,2, \cdots, N\right\}$ in a tensor algebra $\otimes_{i=1}^{N} \mathcal{N}_{i}$

[(ii; b)] Parallel measurement]: A measurement $\mathrm{M}_{\otimes_{i=1}^{N} \mathcal{N}_{i}}\left(\otimes_{i=1}^{N} \mathrm{O}_{i}, S_{\left[\otimes_{i=1}^{N} \rho_{i}\right]}\right)$ is called a parallel measurement concerning $\left\{\mathrm{M}_{\mathcal{N}_{i}}\left(\mathrm{O}_{i}:=\left(X_{i}, \mathcal{F}_{i}, F_{i}\right), S_{\left[\rho_{i}\right]}\right)\right\}_{i=1}^{N}$.

\section{Why Does Logic Arise in Classical QL?}

It is well-known that logic holds in the class of mathematical propositions. However, it should be noted that it is not guaranteed that logic holds among non-mathematical propositions. Thus, the question "Why does logic arise in classical QL?” is significant.

From here, we devote ourselves to classical QL (in the classical basic structure $\left[C_{0}(\Omega) \subseteq L^{\infty}(\Omega, v) \subseteq B\left(L^{2}(\Omega, v)\right)\right]$ ) and not quantum QL (in the quantum basic structure $[\mathcal{C}(H) \subseteq B(H) \subseteq B(H)]$ ). 
The close relationship between measurement and logic was first discussed in ref. [4]. The argument in this section is regarded as a slight variation of the argument in ref. [4].

\subsection{Logic (i.e., ᄀ, $\wedge, \vee, \rightarrow$ ) in Classical QL}

We have the following theorem:

Theorem 5. In a class of binary projective measurements in classical QL, measurement has properties like logic:

In this section we will devote ourselves to the above proof as follows.

Consider a classical basic structure

$$
\left[C_{0}(\Omega) \subseteq L^{\infty}(\Omega, v) \subseteq B\left(L^{2}(\Omega, v)\right)\right]
$$

Here, assume that $\Omega$ is a locally compact space with a Borel measure $v$ on $\Omega$ such that $v(D)>0$ (for any open set $D(\subseteq \Omega, D \neq \varnothing)$. Also, without loss of generality, we assume that $v(\Omega)=1$. Consider many tomatoes, that is, roughly speaking, consider $T$ as the set of all tomatoes. Assume that any tomato $t(\in T)$ is represented by a state $\omega$, which is an element of the state space $\Omega$. Thus, we have the map $\hat{\omega}: T \rightarrow \Omega$. That is, the quantitative property of a tomato $t$ is represented by $\hat{\omega}(t)$. For example, it suffices to consider $\Omega$ such that $\Omega \subseteq \mathbb{R}^{N}$ (=N-dimensional real space), where $N$ is sufficiently large natural number (or, $N=\infty$ ). That is,

$$
\begin{gathered}
\Omega \ni \omega=\left(\omega^{(1)}(=\text { weight }), \omega^{(2)}(=\text { diameter }), \omega^{(3)}(=\text { diameter }),\right. \\
\omega^{(4)}(=\text { color value }), \omega^{(5)}(=\text { calorie }), \\
\left.\omega^{(6)}(=\text { sugar content }), \cdots, \omega^{(N)}(=\cdots)\right) \in \mathbb{R}^{N}
\end{gathered}
$$

Consider a binary projective observable (i.e., $\{1,0\}$-valued projective observable, or $\left\{x_{1}, x_{0}\right\}$-valued projective observable) $\mathrm{O} \equiv\left(X, 2^{X}, F\right)$ in $L^{\infty}(\Omega, v)$, where $X=\{1,0\}$ (or, $X=\left\{x_{1}, x_{0}\right\}$ ) and $F(\Xi)=[F(\Xi)]^{2} \quad(\forall \Xi \in \mathcal{F})$.

Further, as shown in Figure 1 below, we, for the sake of simplicity, assume that $\{\omega \in \Omega \mid[F(\{1\})(\omega)=1$, a.e. $\}(\equiv \Gamma)$ is an open set such that

$$
\Gamma=[\bar{\Gamma}]^{\circ}, \quad v\left(\Omega \backslash\left(\Gamma \cup\left[\Gamma^{c}\right]^{\circ}\right)\right)=0
$$

where $D^{c}$ is the complement of $D$, i.e., $\Omega \backslash D, \bar{D}=$ "the closure of $D^{\prime}, D^{\circ}$

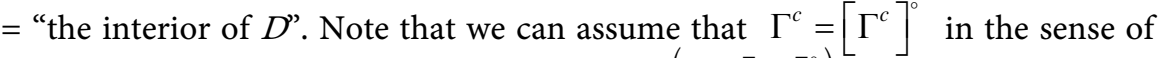
“almost everywhere concerning $v$ " (i.e., “ $v\left(\Gamma^{c} \backslash\left[\Gamma^{c}\right]^{\circ}\right)=0$ ”), which will be

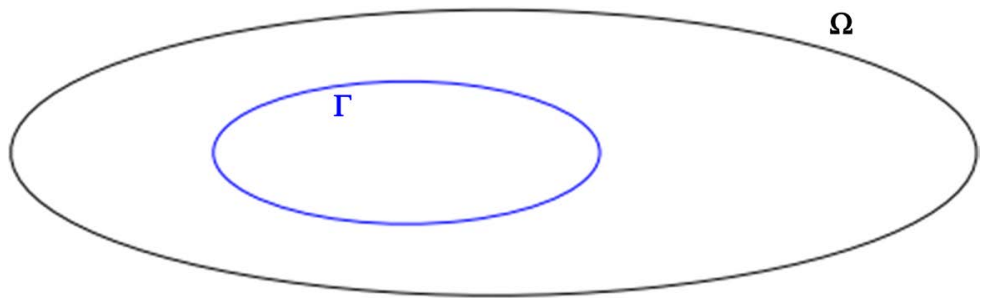

Figure 1. Venn diagram: $\Gamma \subseteq \Omega$. 
frequently used without refusal in this paper. The $\{1,0\}$-valued projective observable $\mathrm{O} \equiv\left(X, 2^{X}, F\right)$ in $L^{\infty}(\Omega, v)$ is also denoted by

$$
\mathrm{O}^{\Gamma} \equiv\left(X, 2^{X}, F^{\Gamma}\right)
$$

Remark 6. (i): Someone might say that the term "the set of all tomatoes" is as ambiguous as "the set of all dinosaurs". However, for the sake of convenience, here we use the term "the set of all tomatoes". This problem is the same as that of the Hempel' raven paradox. For further discussion about this, see ref. [20] [21].

(ii): If we want to both tomato's world $\Omega_{1}$ and apple's world $\Omega_{2}$, it suffices to start from the product space $\Omega_{1} \times \Omega_{2}$. Thus, in general we consider the world also is represented by a large state space $\hat{\Omega}$.

Definition 7. [Measured value] Consider a measurement $\mathrm{M}_{L^{\infty}(\Omega, v)}\left(\mathrm{O}^{\Gamma}, S_{[\omega]}\right)$, where $\mathrm{O}^{\Gamma} \equiv\left(X(=\{1,0\}), 2^{X}, F^{\Gamma}\right)$ is a binary projective observable in $L^{\infty}(\Omega, v)$. Denote the measured value of $\mathrm{M}_{L^{\infty}(\Omega, \nu)}\left(\mathrm{O}^{\Gamma}, S_{[\omega]}\right)$ by

$[\mathrm{MV}]\left(\mathrm{M}_{L^{\infty}(\Omega, v)}\left(\mathrm{O}^{\Gamma}, S_{[\omega]}\right)\right)$. Then, we see that

$$
[\mathbf{M V}]\left(\mathbf{M}_{L^{\infty}(\Omega, v)}\left(\mathrm{O}^{\Gamma}, S_{[\omega]}\right)\right)= \begin{cases}1 & (\omega \in \Gamma) \\ 0 & (\omega \notin \Gamma)\end{cases}
$$

(with probability 1 ).

Let $X=\{1,0\}, \Omega$ and $\hat{\omega}(t)$ be as before. Put $\Gamma=\mathrm{RD}$, or $\Gamma=\mathrm{SW}$ in the formula (2) (see Figure 2 below). Consider a binary projective observables $\mathrm{O}^{\mathrm{RD}} \equiv\left(X(=\{1,0\}), 2^{X}, F^{\mathrm{RD}}\right)$ and $\mathrm{O}^{\mathrm{sw}} \equiv\left(X, 2^{X}, F^{\mathrm{sw}}\right)$ in $L^{\infty}(\Omega, v)$. Consider a measurement $\mathrm{M}_{L^{\infty}(\Omega, v)}\left(\mathrm{O}^{\mathrm{RD}}, S_{[\hat{\omega}(t)]}\right)$. That is, we consider that the following three are equivalent (i.e., Axiom 1 (measurement) says that $\left(\mathrm{C}_{1}\right) \Leftrightarrow\left(\mathrm{C}_{2}\right)$. Also, $\left(C_{3}\right)$ is the expression of $\left(C_{1}\right)$ in ordinary language):

$\left(\mathrm{C}_{1}\right)$ A measured value 1 is obtained by the measurement $\mathrm{M}_{L^{\infty}(\Omega, v)}\left(\mathrm{O}^{\mathrm{RD}}, S_{[\hat{\omega}(t)]}\right)$ (i.e., $\left.[\mathrm{MV}]\left(\mathrm{M}_{L^{\infty}(\Omega, v)}\left(\mathrm{O}^{\mathrm{RD}}, S_{[\hat{\omega}(t)]}\right)\right)=1\right)$ (strictly speaking, the probability that a measured value 1 is obtained by the measurement $\mathrm{M}_{L^{\infty}(\Omega, v)}\left(\mathrm{O}^{\mathrm{RD}}, S_{[\hat{\omega}(t)]}\right)$ is equal to 1 .)

$\left(\mathrm{C}_{2}\right) \hat{\omega}(t) \in \mathrm{RD}\left(\equiv\left\{\omega \in \Omega \mid\left[F^{\mathrm{RD}}(\{1\})(\omega)=1\right\}\right)\right.$

$\left(\mathrm{C}_{3}\right)$ A tomato $t$ is "red".

Similarly, as shown in Figure 2 below, consider a measurement $\mathrm{M}_{L^{\infty}(\Omega, v)}\left(\mathrm{O}^{\mathrm{sw}}, S_{[\hat{\omega}(t)]}\right)$. That is, we consider that the following three are equivalent:

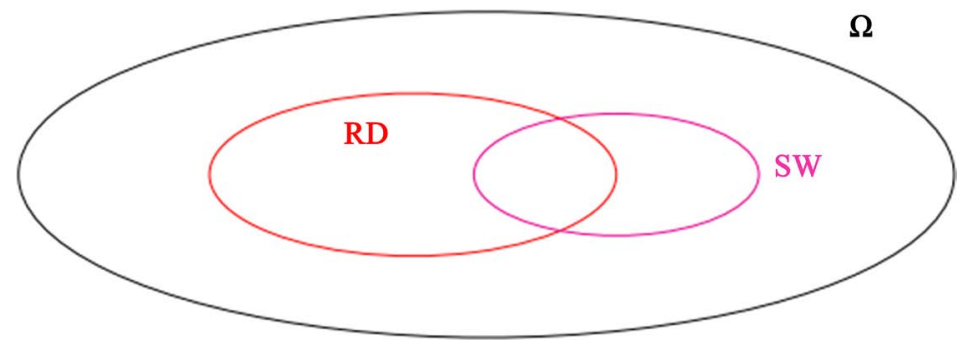

Figure 2. Venn diagram: $\mathrm{RD}, \mathrm{SW} \subseteq \Omega$. 
$\left(\mathrm{C}_{1}^{\prime}\right)$ A measured value 1 is obtained by the measurement

$$
\begin{aligned}
& \mathrm{M}_{L^{\infty}(\Omega, v)}\left(\mathrm{O}^{\mathrm{SW}}, S_{[\hat{\omega}(t)]}\right) \quad\left(\text { i.e., }[\mathbf{M V}]\left(\mathrm{M}_{L^{\infty}(\Omega, v)}\left(\mathrm{O}^{\mathrm{SW}}, S_{[\hat{\omega}(t)]}\right)\right)=1\right) . \\
& \left(\mathrm{C}_{2}^{\prime}\right) \hat{\omega}(t) \in \mathrm{SW}\left(\equiv\left\{\omega \in \Omega \mid\left[F^{\mathrm{SW}}(\{1\})(\omega)=1\right\}\right)\right. \\
& \left(\mathrm{C}_{3}^{\prime}\right) \text { A tomato } t \text { is "sweet". }
\end{aligned}
$$

[Not]

It is clear that the following four are equivalent:

$\left(\mathrm{D}_{0}\right)[\mathbf{M V}]\left(\mathrm{M}_{L^{\infty}(\Omega, v)}\left(\mathrm{O}^{\mathrm{SW}}, S_{[\hat{\omega}(t)]}\right)\right)=0$.

$\left(D_{1}\right)$ A measured value 1 is obtained by the measurement

$\mathrm{M}_{L^{\infty}(\Omega, v)}\left(\Theta_{\neg}\left(\mathrm{O}^{\mathrm{sw}}\right), S_{[\hat{\omega}(t)]}\right)$ (which is also denoted by $\neg \mathrm{M}_{L^{\infty}(\Omega, v)}\left(\mathrm{O}^{\mathrm{sw}}, S_{[\hat{\omega}(t)]}\right)$ ), where $\Theta_{\neg}:\{1,0\} \rightarrow\{1,0\}$ is defined by $\Theta_{\neg}(1)=0, \Theta_{\neg}(0)=1$ (cf. Definition 1; image observable). Thus, $[\mathbf{M V}]\left(\neg \mathrm{M}_{L^{\infty}(\Omega, v)}\left(\mathrm{O}^{\mathrm{sw}}, S_{[\hat{\omega}(t)]}\right)\right)=1$.

$\left(\mathrm{D}_{2}\right) \quad \hat{\omega}(t) \in[\overline{\mathrm{SW}}]^{c}\left(\equiv\left\{\omega \in \Omega \mid\left[F^{\mathrm{SW}}(\{0\})\right](\omega)=0\right\}\right)$

$\left(\mathrm{D}_{3}\right)$ A tomato $t$ is not "sweet".

\section{[And]}

We see that the following four are equivalent:

$\left(\mathrm{E}_{0}\right)$ A measured value $(1,1)$ is obtained by the measurement $\mathrm{M}_{L^{\infty}(\Omega, v)}\left(\mathrm{O}^{\mathrm{sw}} \times \mathrm{O}^{\mathrm{RD}}, S_{[\hat{\omega}(t)]}\right)$.

$\left(\mathrm{E}_{1}\right)$ A measured value 1 is obtained by the measurement $\mathrm{M}_{L^{\infty}(\Omega, v)}\left(\Theta_{\wedge}\left(\mathrm{O}^{\mathrm{SW}} \times \mathrm{O}^{\mathrm{RD}}\right), S_{[\hat{\omega}(t)]}\right)$ (which is also denoted by $\left.\mathrm{M}_{L^{\infty}(\Omega, v)}\left(\mathrm{O}^{\mathrm{SW}}, S_{[\hat{\omega}(t)]}\right) \wedge \mathrm{M}_{L^{\infty}(\Omega, v)}\left(\mathrm{O}^{\mathrm{RD}}, S_{[\hat{\omega}(t)]}\right)\right)$, where $\Theta_{\wedge}:\{1,0\}^{2} \rightarrow\{1,0\}$ is defined by $\Theta_{\wedge}(1,1)=1, \Theta_{\wedge}(1,0)=\Theta_{\wedge}(0,1)=\Theta_{\wedge}(0,0)=0 \quad$ (cf. Definition 1; image observable). Thus,

$$
\begin{gathered}
{[\mathbf{M V}]\left(\mathrm{M}_{L^{\infty}(\Omega, v)}\left(\mathrm{O}^{\mathrm{SW}}, S_{[\hat{\omega}(t)]}\right) \wedge \mathrm{M}_{L^{\infty}(\Omega, v)}\left(\mathrm{O}^{\mathrm{RD}}, S_{[\hat{\omega}(t)]}\right)\right)=1 .} \\
\hat{\omega}(t) \in \mathrm{SW}\left(\equiv\left\{\omega \in \Omega \mid\left[F^{\mathrm{SW}}(\{1\})\right](\omega)=1\right\}\right) \\
\left(\mathrm{E}_{2}\right) \quad \mathrm{RD}\left(\equiv\left\{\omega \in \Omega \mid\left[F^{\mathrm{RD}}(\{1\})\right](\omega)=1\right\}\right)
\end{gathered}
$$

$\left(\mathrm{E}_{3}\right)$ A tomato $t$ is "sweet" and "red"

Remark 8. When $\omega_{1} \neq \omega_{2}$, it should be noted that the simbol “ $\mathrm{M}_{L^{\infty}(\Omega, v)}\left(\mathrm{O}^{\mathrm{SW}}, S_{\left[\omega_{1}\right]}\right) \wedge \mathrm{M}_{L^{\infty}(\Omega, v)}\left(\mathrm{O}^{\mathrm{RD}}, S_{\left[\omega_{2}\right]}\right)$ " is not yet defined. Recall the linguistic Copenhagen interpretation "Only one measurement is permitted". Thus, "there is only one state". Therefore, this should be defined by the parallel $\mathrm{M}_{L^{\infty}(\Omega, v) \otimes L^{\infty}(\Omega, v)}\left(\Theta_{\wedge}\left(\mathrm{O}^{\mathrm{SW}} \otimes \mathrm{O}^{\mathrm{RD}}\right), S_{\left[\left(\omega_{1}, \omega_{2}\right)\right]}\right)$. More generally, the simbol

“ $\wedge_{\lambda \in \Lambda} \mathrm{M}_{L^{\infty}\left(\Omega_{\lambda}, v_{\lambda}\right)}\left(\mathrm{O}^{\Gamma_{\lambda}}, S_{\left[\omega_{\lambda}\right]}\right)$ ” is defined by

$$
\mathrm{M}_{\substack{\otimes \in \Lambda \\ L^{\infty}\left(\Omega_{\lambda}, v_{\lambda}\right)}}\left(\otimes_{\lambda \in \Lambda}^{\otimes} \mathrm{O}^{\Gamma_{\lambda}}, S_{\left[\left(\omega_{\lambda}\right)_{\lambda \in \Lambda}\right]}\right)
$$

[Or]

We see that the following four are equivalent:

$\left(\mathrm{F}_{0}\right)$ A measured value $\left(x_{1}, x_{2}\right)$ obtained by the measurement 
$\mathrm{M}_{L^{\infty}(\Omega, v)}\left(\mathrm{O}^{\mathrm{SW}} \times \mathrm{O}^{\mathrm{RD}}, S_{[\hat{\omega}(t)]}\right)$ belongs to $\{(1,1),(1,0),(0,1)\}$

$\left(\mathrm{F}_{1}\right)$ A measured value 1 is obtained by the measurement

$\mathrm{M}_{L^{\infty}(\Omega, v)}\left(\Theta_{v}\left(\mathrm{O}^{\mathrm{SW}} \times \mathrm{O}^{\mathrm{RD}}\right), S_{[\hat{\omega}(t)]}\right)$ (which is also denoted by

$\left.\mathrm{M}_{L^{\infty}(\Omega, v)}\left(\mathrm{O}^{\mathrm{SW}}, S_{[\hat{\omega}(t)]}\right) \vee \mathrm{M}_{L^{\infty}(\Omega, v)}\left(\mathrm{O}^{\mathrm{RD}}, S_{[\hat{\omega}(t)]}\right)\right)$, where $\Theta_{\vee}:\{1,0\}^{2} \rightarrow\{1,0\}$ is de-

fined by $\Theta_{\vee}(1,1)=\Theta_{\vee}(1,0)=\Theta_{\vee}(0,1)=1, \Theta_{\vee}(0,0)=0$.

Thus, $[\mathbf{M V}]\left(\mathrm{M}_{L^{\infty}(\Omega, v)}\left(\mathrm{O}^{\mathrm{SW}}, S_{[\hat{\omega}(t)]}\right) \vee \mathrm{M}_{L^{\infty}(\Omega, v)}\left(\mathrm{O}^{\mathrm{RD}}, S_{[\hat{\omega}(t)]}\right)\right)=1$.

$\left(\mathrm{F}_{2}\right)$

$$
\begin{aligned}
\hat{\omega}(t) \in \mathrm{SW}( & \left.\equiv\left\{\omega \in \Omega \mid\left[F^{\mathrm{sW}}(\{1\})\right](\omega)=1\right\}\right) \\
& \cup \operatorname{RD}\left(\equiv\left\{\omega \in \Omega \mid\left[F^{\mathrm{RD}}(\{1\})\right](\omega)=1\right\}\right)
\end{aligned}
$$

$\left(\mathrm{F}_{3}\right)$ A tomato $t$ is "sweet" or "red"

\section{[Implication]}

We see that the following four are equivalent:

$\left(\mathrm{G}_{0}\right)$ A measured value $\left(x_{1}, x_{2}\right)$ obtained by the measurement

$\mathrm{M}_{L^{\infty}(\Omega, v)}\left(\mathrm{O}^{\mathrm{SW}} \times \mathrm{O}^{\mathrm{RD}}, S_{[\hat{\omega}(t)]}\right)$ belongs to $\{(1,1),(0,1),(0,0)\}$

$\left(\mathrm{G}_{1}\right)$ A measured value 1 is obtained by the measurement

$\mathrm{M}_{L^{\infty}(\Omega, v)}\left(\Theta_{\rightarrow}\left(\mathrm{O}^{\mathrm{SW}} \times \mathrm{O}^{\mathrm{RD}}\right), S_{[\hat{\omega}(t)]}\right)$ (which is also denoted by

$\left.\mathrm{M}_{L^{\infty}(\Omega, v)}\left(\mathrm{O}^{\mathrm{SW}}, S_{[\hat{\omega}(t)]}\right) \rightarrow \mathrm{M}_{L^{\infty}(\Omega, v)}\left(\mathrm{O}^{\mathrm{RP}}, S_{[\hat{\omega}(t)]}\right)\right)$, where $\Theta_{\rightarrow}:\{1,0\}^{2} \rightarrow\{1,0\}$ is defined by $\Theta_{\rightarrow}(1,1)=\Theta_{\rightarrow}(0,1)=\Theta_{\rightarrow}(0,0)=1 \quad, \quad \Theta_{\rightarrow}(1,0)=0 \quad$. Thus, $[\mathbf{M V}]\left(\mathrm{M}_{L^{\infty}(\Omega, v)}\left(\mathrm{O}^{\mathrm{SW}}, S_{[\hat{\omega}(t)]}\right) \rightarrow \mathrm{M}_{L^{\infty}(\Omega, v)}\left(\mathrm{O}^{\mathrm{RD}}, S_{[\hat{\omega}(t)]}\right)\right)=1$.

$\left(\mathrm{G}_{2}\right)$

$$
\begin{array}{r}
\hat{\omega}(t) \in \mathrm{SW}^{c}\left(\equiv\left\{\omega \in \Omega \mid\left[F^{\mathrm{s \omega}}(\{1\})\right](\omega)=0\right\}\right) \\
\operatorname{URD}\left(\equiv\left\{\omega \in \Omega \mid\left[F^{\mathrm{RD}}(\{1\})\right](\omega)=1\right\}\right)
\end{array}
$$

$\left(\mathrm{G}_{3}\right)$ A tomato $t$ is not "sweet", or it is "red"

Summing up the above, we have the following theorem:

Theorem 9. We see that for each $\omega \in \Omega$,

(a) $[\mathbf{M V}]\left(\neg \mathrm{M}_{L^{\infty}(\Omega, v)}\left(\mathrm{O}^{\Gamma}, S_{[\hat{\omega}(t)]}\right)\right)=1-[\mathbf{M V}]\left(\mathrm{M}_{L^{\infty}(\Omega, v)}\left(\mathrm{O}^{\Gamma}, S_{[\hat{\omega}(t)]}\right)\right)$

(b)

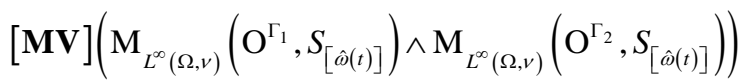

)

$$
=\min \left\{[\mathbf{M V}]\left(\mathbf{M}_{L^{\infty}(\Omega, v)}\left(\mathrm{O}^{\Gamma_{1}}, S_{[\hat{\omega}(t)]}\right)\right),[\mathbf{M V}]\left(\mathbf{M}_{L^{\infty}(\Omega, v)}\left(\mathrm{O}^{\Gamma_{2}}, S_{[\hat{\omega}(t)]}\right)\right)\right\}
$$

(c)

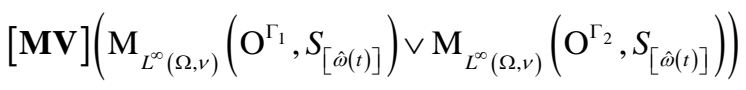

$$
=\max \left\{[\mathbf{M V}]\left(\mathbf{M}_{L^{\infty}(\Omega, v)}\left(\mathrm{O}^{\Gamma_{1}}, S_{[\hat{\omega}(t)]}\right)\right),[\mathbf{M V}]\left(\mathbf{M}_{L^{\infty}(\Omega, v)}\left(\mathrm{O}^{\Gamma_{2}}, S_{[\hat{\omega}(t)]}\right)\right)\right\}
$$

(d)

$$
\begin{aligned}
& {[\mathbf{M V}]\left(\mathbf{M}_{L^{\infty}(\Omega, v)}\left(\mathrm{O}^{\Gamma_{1}}, S_{[\hat{\omega}(t)]}\right) \rightarrow \mathbf{M}_{L^{\infty}(\Omega, v)}\left(\mathrm{O}^{\Gamma_{2}}, S_{[\hat{\omega}(t)]}\right)\right)} \\
& =\max \left\{1-[\mathbf{M V}]\left(\mathbf{M}_{L^{\infty}(\Omega, v)}\left(\mathrm{O}^{\Gamma_{1}}, S_{[\hat{\omega}(t)]}\right)\right),[\mathbf{M V}]\left(\mathbf{M}_{L^{\infty}(\Omega, v)}\left(\mathrm{O}^{\Gamma_{2}}, S_{[\hat{\omega}(t)]}\right)\right)\right\}
\end{aligned}
$$

Therefore, we can expect that a class of classical binary projective measure- 
ments has a logical structure. In this sense, this class can be regarded as a class of "propositions".

Remark 10. Note that propositional logic (i.e., $\neg, \wedge, \vee, \rightarrow$ ) and predict logic (i.e., $\neg, \wedge, \vee, \rightarrow, \forall, \exists$ ) are essentially the same since $P_{1} \wedge P_{2} \wedge P_{3} \wedge \cdots=(\forall n)\left[P_{n}\right]$ and $P_{1} \vee P_{2} \vee P_{3} \vee \cdots=(\exists n)\left[P_{n}\right]$. Thus, this paper does not distinguish propositional logic and predict logic.

\subsection{Syllogism}

Further, consider a measurement $\mathrm{M}_{L^{\infty}(\Omega, v)}\left(\mathrm{O}^{\mathrm{RP}}, S_{[\hat{\omega}(t)]}\right)$. That is, we consider that the following three are equivalent:

$\left(\mathrm{H}_{1}\right)$ A measured value 1 is obtained by the measurement $\mathrm{M}_{L^{\infty}(\Omega, v)}\left(\mathrm{O}^{\mathrm{RP}}, S_{[\hat{\omega}(t)]}\right)$.

$\left(\mathrm{H}_{2}\right) \hat{\omega}(t) \in \mathrm{RP}\left(\equiv\left\{\omega \in \Omega \mid\left[F^{\mathrm{RP}}(\{1\})\right](\omega)=1\right\}\right)$

$\left(\mathrm{H}_{3}\right)$ A tomato $t$ is "ripe".

Theorem 11. [Syllogism]:

Let $t$ be a tomato, and let $\hat{\omega}(t)(\in \Omega)$ be the state of $t$. Assume the followings:

$\left(\mathrm{I}_{0}\right)$ A measured value $\left(x_{1}, x_{2}\right)$ obtained by the measurement $\mathrm{M}_{L^{\infty}(\Omega, v)}\left(\mathrm{O}^{\mathrm{SW}} \times \mathrm{O}^{\mathrm{RP}}, S_{[\hat{\omega}(t)]}\right)$ belongs to $\{(1,1),(0,1),(0,0)\}$

which is equivalent to

$\left(\mathrm{I}_{1}\right)[\mathbf{M V}]\left(\mathrm{M}_{L^{\infty}(\Omega, v)}\left(\mathrm{O}^{\mathrm{SW}}, S_{[\hat{\omega}(t)]}\right) \rightarrow \mathrm{M}_{L^{\infty}(\Omega, v)}\left(\mathrm{O}^{\mathrm{RP}}, S_{[\hat{\omega}(t)]}\right)\right)=1$

$\left(\mathrm{I}_{2}\right)$

$$
\begin{aligned}
& \hat{\omega}(t) \notin \mathrm{SW}\left(\equiv\left\{\omega \in \Omega \mid\left[F^{\mathrm{SW}}(\{1\})\right](\omega)=1\right\}\right) \\
& \vee \hat{\omega}(t) \in \operatorname{RP}\left(\equiv\left\{\omega \in \Omega \mid\left[F^{\mathrm{RP}}(\{1\})\right](\omega)=1\right\}\right)
\end{aligned}
$$

$\left(\mathrm{I}_{3}\right)$ A tomato $t$ is not "sweet", or it is "ripe". and

( $\left.\mathrm{I}_{0}^{\prime}\right)$ A measured value $\left(x_{2}, x_{3}\right)$ obtained by the measurement $\mathrm{M}_{L^{\infty}(\Omega, v)}\left(\mathrm{O}^{\mathrm{RP}} \times \mathrm{O}^{\mathrm{RD}}, S_{[\hat{\omega}(t)]}\right)$ belongs to $\{(1,1),(0,1),(0,0)\}$

which is equivalent to

$$
\left(\mathrm{I}_{1}^{\prime}\right)[\mathbf{M V}]\left(\mathrm{M}_{L^{\infty}(\Omega, v)}\left(\mathrm{O}^{\mathrm{RP}}, S_{[\hat{\omega}(t)]}\right) \rightarrow \mathrm{M}_{L^{\infty}(\Omega, v)}\left(\mathrm{O}^{\mathrm{RD}}, S_{[\hat{\omega}(t)]}\right)\right)=1
$$

which is equivalent to

$$
\begin{aligned}
& \left(\mathrm{I}_{2}^{\prime}\right) \hat{\omega}(t) \notin \mathrm{RP}\left(\equiv\left\{\omega \in \Omega \mid\left[F^{\mathrm{RP}}(\{1\})\right](\omega)=1\right\}\right) \\
& \vee \hat{\omega}(t) \in \mathrm{RD}\left(\equiv\left\{\omega \in \Omega \mid\left[F^{\mathrm{RD}}(\{1\})\right](\omega)=1\right\}\right)
\end{aligned}
$$

$\left(\mathrm{I}_{3}^{\prime}\right)$ A tomato $t$ is not "ripe", or it is "red".

Then the following holds:

$\left(\mathrm{J}_{0}\right)$ A measured value $\left(x_{1}, x_{3}\right)$ obtained by the measurement $\mathrm{M}_{L^{\infty}(\Omega, v)}\left(\mathrm{O}^{\mathrm{SW}} \times \mathrm{O}^{\mathrm{RD}}, S_{[\hat{\omega}(t)]}\right)$ belongs to $\{(1,1),(0,1),(0,0)\}$

$\left(\mathrm{J}_{1}\right)[\mathbf{M V}]\left(\mathrm{M}_{L^{\infty}(\Omega, v)}\left(\mathrm{O}^{\mathrm{SW}}, S_{[\hat{\omega}(t)]}\right) \rightarrow \mathrm{M}_{L^{\infty}(\Omega, v)}\left(\mathrm{O}^{\mathrm{RD}}, S_{[\hat{\omega}(t)]}\right)\right)=1$

$\left(\mathrm{J}_{2}\right)$

$$
\begin{aligned}
& \hat{\omega}(t) \notin \mathrm{SW}\left(\equiv\left\{\omega \in \Omega \mid\left[F^{\mathrm{RD}}(\{1\})\right](\omega)=1\right\}\right) \\
& \vee \hat{\omega}(t) \in \mathrm{RD}\left(\equiv\left\{\omega \in \Omega \mid\left[F^{\mathrm{RP}}(\{1\})\right](\omega)=1\right\}\right)
\end{aligned}
$$


$\left(\mathrm{J}_{3}\right)$ A tomato $t$ is not "sweet", or it is "red".

[Proof]: Recalling the linguistic Copenhagen interpretation "Only one measurement is permitted" (in Sec. 2.3), we have enough to see the simultaneous observable $\mathrm{O}^{\mathrm{SW}} \times \mathrm{O}^{\mathrm{RP}} \times \mathrm{O}^{\mathrm{RD}}$, which uniquely exists (cf. Definition 4 (i)). Thus, we have the measurement $\mathrm{M}_{L^{\infty}(\Omega, v)}\left(\mathrm{O}^{\mathrm{SW}} \times \mathrm{O}^{\mathrm{RP}} \times \mathrm{O}^{\mathrm{RD}}, S_{[\hat{\omega}(t)]}\right)$. Let $\left(x_{1}, x_{2}, x_{3}\right)$ be the measured value. We easily see that $\left(x_{1}, x_{3}\right)$ belongs to $\{(1,1),(0,1),(0,0)\}$. Thus, (J) holds.

However, we should add the following. This proof is not self-evident since the existence and uniqueness of the simultaneous observable $\mathrm{O}^{\mathrm{SW}} \times \mathrm{O}^{\mathrm{RP}} \times \mathrm{O}^{\mathrm{RD}}$ is not trivial (cf. Definition 4 (i)). Also, see Sec. 5 (i.e., Syllogizm does not always hold in quantum sistems).

\subsection{Elementary Measurements}

Consider the state space $\Omega$, which is finite (or, countable) with a metric $d$ (i.e., $d\left(\omega_{1}, \omega_{2}\right)=1\left(\omega_{1} \neq \omega_{2}\right), \quad=0\left(\omega_{1}=\omega_{2}\right)$.

Definition 12. Let $\lambda$ be any element of $\Omega$. Putting $\Gamma=\{\lambda\}$ in the formula (2), define the elementary binary projective observable

$$
\begin{aligned}
& \mathrm{O}^{\{\lambda\}}=\left(X(=\{1,0\}), 2^{X}, F^{\{\lambda\}}\right) \text { in } L^{\infty}(\Omega, v) \text { such that } \\
& {\left[F^{\{\lambda\}}(\{1\})\right](\omega)= \begin{cases}1 & (\text { if } \omega=\lambda) \\
0 & \text { if } \omega \neq \lambda)\end{cases} } \\
& {\left[F^{\{\lambda\}}(\{0\})\right](\omega)=1-\left[F_{\{\lambda\}}(\{1\})\right](\omega) \quad(\forall \omega \in \Omega) }
\end{aligned}
$$

The measurement $\mathrm{M}_{L^{\infty}(\Omega, v)}\left(\mathrm{O}^{\{\lambda\}}, S_{[\omega]}\right)(\lambda, \omega \in \Omega)$ is called an elementary measurement.

It is clear that it holds that

$\left(\mathrm{K}_{1}\right)$ A measured value 1 is obtained by the elementary measurement $\mathrm{M}_{L^{\infty}(\Omega, v)}\left(\mathrm{O}^{\{\lambda\}}, S_{[\omega]}\right) \Leftrightarrow \lambda=\omega$

$\left(\mathrm{K}_{2}\right)$ A measured value 1 is obtained by the elementary measurement $\mathrm{M}_{L^{\infty}(\Omega, v)}\left(\mathrm{O}^{\{\lambda\}}, S_{[\omega]}\right) \Leftrightarrow \lambda \neq \omega$

Under the above preparation, the following theorem is clear

Theorem 13. Let $\Gamma$ be a subset of $\Omega$. And let $\omega \in \Omega$. Then we see that

$$
\mathbf{M}_{L^{\infty}(\Omega, v)}\left(\mathrm{O}^{\Gamma}, S_{[\omega]}\right)=\vee_{\lambda \in \Gamma} \mathbf{M}_{L^{\infty}(\Omega, v)}\left(\mathrm{O}^{\{\lambda\}}, S_{[\omega]}\right)
$$

Remark 14. Many readers may not consider the above theorem to be particularly important. I have the same view. However, this theorem was prepared in preparation for Sec.4 (Theorem 23). Of course, the spirit of expressing complex observables in simple observables is quite important. In quantum language, this spirit is realized by von Neumann's spectral decomposition theorem (see the formula (3), or [22]) and Holevo's theorem (cf. ref. [26]), that is, "Any observable can be represented by the product of commutative binary projective observables in QL (i.e., both classical and quantum QL)",

Remark 15. In this section, I devoted myself to the classical cases. Our arguments in this section are invalid in quantum cases. Consider a binary projective 
observable $\mathrm{O}=\left(\{1,0\}, 2^{\{1,0\}}, F\right)$ in $B(H)$, where $F(\{1\})=P$ (= projection), $F(\{0\})=I-P$. Define the state $\rho$ by $|e\rangle\langle e|$ (where $e \in H$ such that $\left.\|e\|_{H}=1\right)$. Axiom 1 say that

- The probability that a measured value $1(\in X=\{1,0\})$ is obtained by the measurement $\mathrm{M}\left(\mathrm{O}:=\left(X, 2^{\{1,0\}}, F\right), S_{[\rho]}\right)$ is given by $\operatorname{Tr}[\rho F(\{1\})]\left(=\|P e\|_{H}\right)$

Thus, if $e \notin P H \cup(1-P) H$ (i.e., in most case) the "probability" belongs to the open interval $(0,1)$. That is, in quantum cases, the probability concept cannot be covered up. Thus, the arguments in this section are invalid in quantum cases.

\section{My Understanding of Wittgenstein's Picture Theory}

In Wittgenstein's book “Tractatus Logico-Philosophicus" (cf. [1]), he studies the following:

$\left(\mathrm{L}_{1}\right)$ Assume a certain language $L$. Then, the following problems are essential:

(i) What is a proposition in the language $L$ ? Or, why does logic (i.e., $\neg, \wedge$, $\checkmark, \cdots)$ arise in the language $L$ ?

(ii) Everyone knows that complex propositions can be created by logically combining simple propositions. Now, let's think about the opposite. Is there a class of "simplest propositions (or, elementary propositions)" in the language $L$ ? Or, can any proposition be constructed from elementary propositions?

I think that the above is quite important. However, Wittgenstein's answer is not sufficient, since he did not answer "What is the language $L$ ?"

In the preface of Wittgenstein's book "Tractatus Logico-Philosophicus" (cf. [1]), he said that

$\left(\mathrm{L}_{2}\right)$ This book will perhaps only be understood by those who have themselves already thought the thoughts which are expressed in it-or similar thoughts.

This is a very significant sentence for me. That is because, as I answered in the

Table 1. Logic in quantum language (Sec. 3) vs. logic in Wittgenstein's picture theory (this section).

\begin{tabular}{|c|c|}
\hline Logic in quantum language (Sec. 3 ) & Logic in Wittgenstein's theory (Sec. 4) \\
\hline $\begin{array}{l}\text { Axiom } 1 \text { in Section } 2.2 \text { (what is a measurement?) } \\
\text { the linguistic Copenhagen interpretation in Sec. } 2.3\end{array}$ & $\begin{array}{l}\text { Definition } 17 \text { (what is a proposition?) Naive } \\
\text { set theory ( } \approx \text { Venn diagram: Figure } 3 \text { ) }\end{array}$ \\
\hline system, particle, object, tomato & object, thing, tomato \\
\hline state space (state) & logical space (case, fact,, atomic fact) \\
\hline$[\mathrm{MV}]$, measured value $\{1,0\}$ & {$[\mathrm{TV}]$, truth value $\{\mathrm{T}, \mathrm{F}\}$} \\
\hline classical binary projective measurement & proposition \\
\hline $\mathrm{M}_{L^{\infty}(\Omega, v)}\left(\mathrm{O}^{\Gamma} \equiv\left(X(=\{1,0\}), 2^{X}, F^{\Gamma}\right), S_{[\hat{\omega}(t)]}\right)$ & $\mathrm{P}_{\Omega}\left(\Gamma, S_{[\hat{\omega}(t)]}\right)$ \\
\hline Theorem 11 (Syllogism in measurements) & Theorem 19 (Syllogism in propositions) \\
\hline elementary measurement & elementary proposition \\
\hline Theorem 13 (Remark 14) & Theorem 23 (Remark 24) \\
\hline Elementary measurements are not fundamental & Elementary propositions are fundamental \\
\hline
\end{tabular}


previous section 3, I already know the answers to question $\left(\mathrm{L}_{1}\right)$ before reading this TLP (if the language $L=$ classical QL).

In this section, I propose my understanding of Wittgenstein's picture theory, which is inspired from the arguments in the previous section. The above table will promote the reader's understanding of the arguments in this section.

I encourage you to read the following, referring to the table. If you understand Sec. 3, you should be able to understand this section immediately. Using the above table, we can translate the language of Wittgenstein's theory into quantum language.

\subsection{Logic (i.e., ᄀ, $\wedge, \vee, \rightarrow$ ) in My Understanding of Wittgenstein's Picture Theory}

The question "What is a proposition?" is easy in mathematics. However, outside of mathematics, this question is not easy. Wittgenstein's purpose is to clarify "proposition" in science.

Let $\Omega, \Gamma, \omega, \hat{\omega}(t), \mathrm{SW}, \mathrm{RP}, \mathrm{RD}, \cdots$ be the same as in the previous section. Let us start from the following definition.

Definition 17. [Proposition, Truth value] A pair $(\Gamma, \omega)(\Gamma \subseteq \Omega, \omega \in \Omega)$ is called a proposition in $\Omega$ (i.e., "a system with a state $\omega$ has $\Gamma$-property"), which is denoted by $\mathrm{P}_{\Omega}\left(\Gamma, S_{[\omega]}\right)$. Define the truth value of the proposition $\mathrm{P}_{\Omega}\left(\Gamma, S_{[\omega]}\right)$ (which is denoted by $[\mathbf{T V}]\left(\mathrm{P}_{\Omega}\left(\Gamma, S_{[\omega]}\right)\right)$ ) by

$$
[\mathbf{T V}]\left(\mathrm{P}_{\Omega}\left(\Gamma, S_{[\omega]}\right)\right)= \begin{cases}\mathrm{T}(\text { true }) & (\text { if } \omega \in \Gamma) \\ \mathrm{F}(\text { false }) & (\text { if } \omega \notin \Gamma)\end{cases}
$$

That is, truth values $\{\mathrm{T}, \mathrm{F}\}(=X)$ correspond to measured values $\{1,0\}(=X)$ (or, $X=\left\{x_{1}, x_{0}\right\}$ ). Thus, $\mathrm{I}$ think that the above is essentially the same as naivie set theory (cf. ref. [28]).

If $[\mathbf{T V}]\left(\mathrm{P}_{\Omega}\left(\Gamma, S_{[\hat{\omega}(t)]}\right)\right)=$ "T", we may say that

(M) a tomato $t$ has $\Gamma$-property.

As shown in Figure 3 below, consider a proposition $\mathrm{P}_{\Omega}\left(\mathrm{RD}, S_{[\hat{\omega}(t)]}\right)$. Thus, the following three are equivalent (i.e., $\left(\mathrm{N}_{1}\right) \Leftrightarrow\left(\mathrm{N}_{2}\right)$. Also, $\left(\mathrm{N}_{3}\right)$ is the expression of $\left(\mathrm{N}_{1}\right)$ in ordinary language):

$\left(\mathrm{N}_{1}\right)[\mathbf{T V}]\left(\mathrm{P}_{\Omega}\left(\mathrm{RD}, S_{[\hat{\omega}(t)]}\right)\right)=$ “T”.

$\left(\mathrm{N}_{2}\right) \hat{\omega}(t) \in \mathrm{RD}$

$\left(\mathrm{N}_{3}\right)$ A tomato $t$ is "red".

Similarly, consider a proposition $\mathrm{P}_{\Omega}\left(\mathrm{SW}, S_{[\hat{\omega}(t)]}\right)$. Thus, we see that the following three are equivalent:

$\left(\mathrm{O}_{1}\right)[\mathbf{T V}]\left(\mathrm{P}_{\Omega}\left(\mathrm{SW}, \mathrm{S}_{[\hat{\omega}(t)]}\right)\right)=$ “T”.

$\left(\mathrm{O}_{2}\right) \hat{\omega}(t) \in \mathrm{SW}$

$\left(\mathrm{O}_{3}\right)$ A tomato $t$ is "sweet".

[Not]

It is clear that the following three are equivalent: 


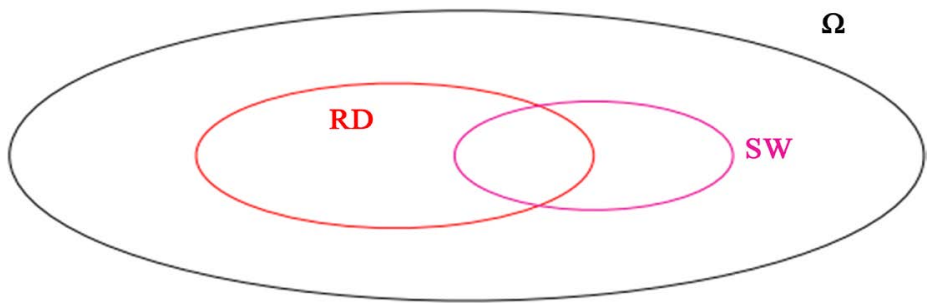

Figure 3. (=Figure 2) Venn diagram: $\mathrm{RD}, \mathrm{SW} \subseteq \Omega$.

$\left(\mathrm{R}_{1}\right)[\mathbf{T V}]\left(\mathrm{P}_{\Omega}\left(\mathrm{SW}^{c}, \mathrm{~S}_{[\hat{\omega}(t)]}\right)\right)=$ "T". ( Here, $\mathrm{P}_{\Omega}\left(\mathrm{SW}^{c}, S_{[\hat{\omega}(t)]}\right)$ is also denoted by $\left.\neg \mathrm{P}_{\Omega}\left(\mathrm{SW}, \mathrm{S}_{[\hat{\omega}(t)]}\right)\right)$, Thus, $[\mathbf{T V}]\left(\neg \mathrm{P}_{\Omega}\left(\mathrm{SW}, \mathrm{S}_{[\hat{\omega}(t)]}\right)\right)=$ "T".

$\left(\mathrm{R}_{2}\right) \quad \hat{\omega}(t) \in[\mathrm{SW}]^{c}$

$\left(\mathrm{R}_{3}\right)$ A tomato $t$ is not "sweet".

[And]

We see that the following three are equivalent:

$\left(\mathrm{S}_{1}\right)$ A truth value of the proposition $\mathrm{P}_{\Omega}\left(\mathrm{SW} \cap \mathrm{RD}, S_{[\hat{\omega}(t)]}\right)$ is equal to "T" (which is also denoted by $\mathrm{P}_{\Omega}\left(\mathrm{SW}, S_{[\hat{\omega}(t)]}\right) \wedge \mathrm{P}_{\Omega}\left(\mathrm{RD}, S_{[\hat{\omega}(t)]}\right)$ ).

Thus, $[\mathbf{T V}]\left(\mathrm{P}_{\Omega}\left(\mathrm{SW}, S_{[\hat{\omega}(t)]}\right) \wedge \mathrm{P}_{\Omega}\left(\mathrm{RD}, S_{[\hat{\omega}(t)]}\right)\right)=$ “T”.

$\left(\mathrm{S}_{2}\right) \quad \hat{\omega}(t) \in \mathrm{SW} \cap \mathrm{RD}$

$\left(\mathrm{S}_{3}\right)$ A tomato $t$ is "sweet" and "red"

[Or]

We see that the following three are equivalent:

$\left(\mathrm{T}_{1}\right)$ A truth value of the proposition $\mathrm{P}_{\Omega}\left(\Theta_{\vee}(\mathrm{SW} \cup \mathrm{RD}), S_{[\hat{\omega}(t)]}\right)$ (which is also denoted by $\left.\mathrm{P}_{\Omega}\left(\mathrm{SW}, S_{[\hat{\omega}(t)]}\right) \vee \mathrm{P}_{\Omega}\left(\mathrm{RD}, S_{[\hat{\omega}(t)]}\right)\right)$ is equal to "T") That is, $[\mathbf{T V}]\left(\mathrm{P}_{\Omega}\left(\mathrm{SW}, S_{[\hat{\omega}(t)]}\right) \vee \mathrm{P}_{\Omega}\left(\mathrm{RD}, S_{[\hat{\omega}(t)]}\right)\right)=$ “T”.

$\left(\mathrm{T}_{2}\right) \hat{\omega}(t) \in \mathrm{SW} \cup \mathrm{RD}$

$\left(\mathrm{T}_{3}\right)$ A tomato $t$ is "sweet" or "red"

\section{[Implication]}

We see that the following three are equivalent:

$\left(\mathrm{U}_{1}\right)$ A truth value of the proposition $\mathrm{P}_{\Omega}\left(\mathrm{SW}^{c} \cup \mathrm{RD}, S_{[\hat{\omega}(t)]}\right)$ (which is also denoted by $\left.\mathrm{P}_{\Omega}\left(\mathrm{SW}, \mathrm{S}_{[\hat{\omega}(t)]}\right) \rightarrow \mathrm{P}_{\Omega}\left(\mathrm{RP}, S_{[\hat{\omega}(t)]}\right)\right)$ is equal to "T", That is, $[\mathbf{T V}]\left(\mathrm{P}_{\Omega}\left(\mathrm{SW}, \mathrm{S}_{[\hat{\omega}(t)]}\right) \rightarrow \mathrm{P}_{\Omega}\left(\mathrm{RD}, S_{[\hat{\omega}(t)]}\right)\right)=$ "T".

$\left(\mathrm{U}_{2}\right) \hat{\omega}(t) \in \mathrm{SW}^{c} \cup \mathrm{RD}$

$\left(\mathrm{U}_{3}\right)$ a tomato $t$ is not "sweet", or it is "red"

Remark 18. Note that the above is essentially the same as logical operation (Boolean algebra; $[\neg, \wedge, \vee, \rightarrow]$ ). However, it should be noted that Definition 17 is essential to the above argument. 


\subsection{Syllogism}

Further, consider a proposition $\mathrm{P}_{\Omega}\left(\mathrm{RP}, \mathrm{S}_{[\hat{\omega}(t)]}\right)$. That is, we consider that the following three are equivalent:

$\left(\mathrm{V}_{1}\right)[\mathbf{T V}]\left(\mathrm{P}_{\Omega}\left(\mathrm{RP}, S_{[\hat{\omega}(t)]}\right)\right)=$ “T”.

$\left(\mathrm{V}_{2}\right) \hat{\omega}(t) \in \mathrm{RP}$

$\left(\mathrm{V}_{3}\right)$ A tomato $t$ is "ripe".

Theorem 19. [Syllogism]:

Let $t$ be a tomato, and let $\hat{\omega}(t)(\in \Omega)$ be the state of $t$. Assume the followings: $\left(\mathrm{W}_{1}\right)[\mathbf{T V}]\left(\mathrm{P}_{\Omega}\left(\mathrm{SW}, \mathrm{S}_{[\hat{\omega}(t)]}\right) \rightarrow \mathrm{P}_{\Omega}\left(\mathrm{RP}, S_{[\hat{\omega}(t)]}\right)\right)=$ “T”.

which is equivalent to

$\left(\mathrm{W}_{2}\right)[\hat{\omega}(t) \notin \mathrm{SW}] \vee[\hat{\omega}(t) \in \mathrm{RP}]$

$\left(\mathrm{W}_{3}\right)$ A tomato $t$ is not "sweet", or it is "ripe".

And further, assume

$\left(\mathrm{W}_{1}^{\prime}\right)[\mathrm{TV}]\left(\mathrm{P}_{\Omega}\left(\mathrm{RP}, S_{[\hat{\omega}(t)]}\right) \rightarrow \mathrm{P}_{\Omega}\left(\mathrm{RD}, S_{[\hat{\omega}(t)]}\right)\right)=$ “T”.

which is equivalent to

$\left(\mathrm{W}_{2}^{\prime}\right)[\hat{\omega}(t) \notin \mathrm{RP}] \vee[\hat{\omega}(t) \in \mathrm{RD}]$

$\left(\mathrm{W}_{3}^{\prime}\right)$ A tomato $t$ is not "ripe", or it is "red".

Then the following holds:

$\left(\mathrm{X}_{1}\right)[\mathbf{T V}]\left(\mathrm{P}_{\Omega}\left(\mathrm{SW}, S_{[\hat{\omega}(t)]}\right) \rightarrow \mathrm{P}_{\Omega}\left(\mathrm{RD}, S_{[\hat{\omega}(t)]}\right)\right)=$ “T”.

$\left(\mathrm{X}_{2}\right)[\hat{\omega}(t) \notin \mathrm{SW}] \vee[\hat{\omega}(t) \in \mathrm{RD}]$

$\left(\mathrm{X}_{3}\right)$ A tomato $t$ is not "sweet", or it is "red".

[Proof using Definition 17]

A simple calculation shows that

$$
\begin{aligned}
& {\left[\mathrm{SW}^{c} \cup \mathrm{RP}\right] \cap\left[\mathrm{PR}^{c} \cup \mathrm{RD}\right]} \\
& =\left[\mathrm{SW}^{c} \cap \mathrm{PR}^{c}\right] \mathrm{U}\left[\mathrm{SW}^{c} \cap \mathrm{RD}\right] \mathrm{U}\left[\mathrm{RP} \cap \mathrm{PR}^{c}\right] \mathrm{U}[\mathrm{RP} \cap \mathrm{RD}] \\
& =\left[\mathrm{SW}^{c} \cap \mathrm{PR}^{c}\right] \cup\left[\mathrm{SW}^{c} \cap \mathrm{RD}\right] \mathrm{U}[\mathrm{RP} \cap \mathrm{RD}] \subseteq \mathrm{SW}^{c} \cup \mathrm{RD}
\end{aligned}
$$

Recalling $\left(\mathrm{W}_{2}\right)$ and $\left(\mathrm{W}_{2}^{\prime}\right)$, we immediately see $\left(\mathrm{X}_{2}\right)$. and thus, $\left(\mathrm{X}_{1}\right),\left(\mathrm{X}_{3}\right)$.

Remark 20. Note that the proof of this theorem (due to Definition 17) is simple compared to Theorem 11 (using Axiom 1 and the linguistic Copenhagen interpretation in Sec. 2.3). That is, in the proof of Theorem 19 we do not need to check the existence and uniqueness of the simultaneous observable $\mathrm{O}^{\mathrm{SW}} \times \mathrm{O}^{\mathrm{RP}} \times \mathrm{O}^{\mathrm{RD}}$.

The following exercise will promote the reader's understanding of "proposition".

Exercise 21. Let $\Gamma \subseteq \Omega$ and $\Lambda \subseteq \Omega$. Then, we have the following question.

- Is the statement " $\Lambda \subseteq \Gamma$ " a proposition?

[Answer]:

$$
\begin{aligned}
& \Lambda \subseteq \Gamma \\
& \Leftrightarrow(\forall \lambda \in \Lambda)[\lambda \in \Gamma] \\
& \Leftrightarrow\left(\omega_{\lambda}\right)_{\lambda \in \Lambda} \in \underset{\lambda \in \Lambda}{\times} \Gamma_{\lambda} \quad\left(\text { where } \omega_{\lambda}:=\lambda(\forall \lambda \in \Lambda)\right), \Gamma_{\lambda}:=\Gamma(\forall \lambda \in \Lambda)
\end{aligned}
$$




$$
\Leftrightarrow \mathrm{P}_{\Omega^{\Lambda}}\left[\underset{\lambda \in \Lambda}{\times} \Gamma_{\lambda}, S_{\left[\left(\omega_{\lambda}\right)_{\lambda \in \Lambda}\right]}\right] \quad \text { (where } \Omega^{\Lambda} \text { is } \Lambda \text {-dimensional product space) }
$$

Thus, the statement " $\Lambda \subseteq \Gamma$ " is a proposition in $\Omega^{\Lambda}$.

\subsection{Elementary Propositions}

Consider the state space $\Omega$, which is finite (or, countable) with a metric $d$ (i.e., $d\left(\omega_{1}, \omega_{2}\right)=1\left(\omega_{1} \neq \omega_{2}\right),=0\left(\omega_{1}=\omega_{2}\right)$. Further, assume that the Borel measure $v$ is defined by the point measure, i.e., $v(\{\omega\})=1(\forall \omega \in \Omega)$.

Definition 22. Let $\lambda$ be any element of $\Omega$. Putting $\Gamma=\{\lambda\}$, define the proposition $\mathrm{P}_{\Omega}\left(\{\lambda\}, S_{[\omega]}\right)$, which is called an elementary proposition.

It is clear that it holds that

- A truth value of the elementary proposition $\mathrm{P}_{\Omega}\left(\{\lambda\}, S_{[\omega]}\right)$ is equal to "T" $\Leftrightarrow \lambda=\omega$

- A truth value of the elementary proposition $\mathrm{P}_{\Omega}\left(\{\lambda\}, S_{[\omega]}\right)$ is equal to "F" $\Leftrightarrow \lambda \neq \omega$

Theorem 23. Let $\Gamma$ be a subset of $\Omega$. And let $\omega \in \Omega$. Then we see that

$$
\mathrm{P}_{\Omega}\left(\Gamma, S_{[\omega]}\right)=\vee_{\lambda \in \Gamma} \mathrm{P}_{\Omega}\left(\{\lambda\}, S_{[\omega]}\right)
$$

That is, every proposition can be represented by the sum of elementary propositions. This is not trivial since Exercise 21 is not trivial.

[Proof].

We see that

The true value of $\mathrm{P}_{\Omega}\left(\Gamma, S_{[\omega]}\right)$ is equal to "T"

$$
\begin{aligned}
& \Leftrightarrow \omega \in \Gamma \\
& \Leftrightarrow \exists \lambda(\in \Gamma)[\omega \in\{\lambda\}] \\
& \Leftrightarrow \text { The true value of } \vee_{\lambda \in \Gamma} \mathrm{P}_{\Omega}\left(\{\lambda\}, S_{[\omega]}\right) \text { is equal to "T" }
\end{aligned}
$$

Remark 24. I believe that the above is the main assertion in Wittgenstein's picture theory. However many readers may not consider the above theorem to be particularly important. I have the same view. In fact, this theorem holds only in special cases such as $\Omega$ is a finite (or $\Omega$ has a discrete topology).

\section{Syllogism Does Not Always Hold in Quantum Systems}

I was devoted to classical systems in Sections 3 and 4. In this section, I note the fact such that

(Y) syllogism does not always hold in quantum systems.

Although this $(\mathrm{Y})$ is not main in this paper, this will promote the understanding of "logic" in QL.

Let us prove it as follows. The proof is a slightly modification of the argument about EPR-paradox and Heisenberg's uncertainty relation (cf. [29] [30]).

Note that an observable $\mathrm{O}=(\mathbb{R}, \mathcal{B}, F)$ in $B(H)$ can be identified with a self-adjoint operator $L$ on a Hilbert space $H$ such as 


$$
\begin{aligned}
& \mathrm{O}=(\mathbb{R}, \mathcal{B}, F) \longleftrightarrow \text { identification } \\
& L=\int_{\mathbb{R}} \lambda F(\mathrm{~d} \lambda):(\text { spectral decomposition theorem })
\end{aligned}
$$

(cf. [22] [9]). Thus we mainly use self-adjoint operator representations below.

A quantum two particles system $S$ is formulated in a tensor Hilbert space $H=H_{1} \otimes H_{1}=L^{2}\left(\mathbb{R}_{q_{1}}\right) \otimes L^{2}\left(\mathbb{R}_{q_{2}}\right)=L^{2}\left(\mathbb{R}_{\left(q_{1}, q_{2}\right)}^{2}\right)$. The state

$u_{0}\left(\in H=H_{1} \otimes H_{1}=L^{2}\left(\mathbb{R}_{\left(q_{1}, q_{2}\right)}^{2}\right)\right)$ (or precisely, $\rho_{0}=\left|u_{0}\right\rangle\left\langle u_{0}\right|$ ) of the system $S$ is assumed to be

$$
u_{0}\left(q_{1}, q_{2}\right)=\sqrt{\frac{1}{2 \pi \epsilon \sigma}} \exp \left[-\frac{1}{8 \epsilon^{2}}\left(q_{1}-q_{2}+a\right)^{2}-\frac{1}{8 \sigma^{2}}\left(q_{1}+q_{2}\right)^{2}\right]
$$

where $a \neq 0$, a positive number $\epsilon$ is sufficiently small, and a positive number $\sigma$ is sufficiently large. Thus, we see that

$$
\left|\widehat{u_{0}}\left(p_{1}, q_{2}\right)\right|=\left|\sqrt{\frac{1}{2 \pi \epsilon \sigma}} \exp \left[-\frac{1}{8 \sigma^{2}}\left(p_{1}-p_{2}\right)^{2}-\frac{1}{8 \epsilon^{2}}\left(p_{1}+p_{2}\right)^{2}\right]\right|
$$

where $\widehat{u_{0}}$ is the Fourier transform of $u_{0}$.

For each $k=1,2$, define the self-adjoint operators

$$
\begin{gathered}
Q_{k}: L^{2}\left(\mathbb{R}_{\left(q_{1}, q_{2}\right)}^{2}\right) \rightarrow L^{2}\left(\mathbb{R}_{\left(q_{1}, q_{2}\right)}^{2}\right) \text { and } P_{k}: L^{2}\left(\mathbb{R}_{\left(q_{1}, q_{2}\right)}^{2}\right) \rightarrow L^{2}\left(\mathbb{R}_{\left(q_{1}, q_{2}\right)}^{2}\right) \text { by } \\
Q_{1}=q_{1}, \quad P_{1}=\frac{\hbar \partial}{i \partial q_{1}} \\
Q_{2}=q_{2}, \quad P_{2}=\frac{\hbar \partial}{i \partial q_{2}}
\end{gathered}
$$

where we assume, for simplicity, that $\hbar=1$.

Thus, we see:

$\left(\mathrm{Z}_{1}\right)$ Let $\mathrm{O}_{1}=\left(\mathbb{R}^{3}, \mathcal{B}_{\mathbb{R}^{3}}, F_{1}\right)$ in $B(H)\left(=B\left(L^{2}\left(\mathbb{R}^{2}\right)\right)\right)$ be the observable representation of the self-adjoint operator $\left(Q_{1} \otimes P_{2}\right) \times\left(I \otimes P_{2}\right)$. And consider the measurement $\mathrm{M}_{B(H)}\left(\mathrm{O}_{1}=\left(\mathbb{R}^{3}, \mathcal{B}_{\mathbb{R}^{3}}, F_{1}\right), S_{\left[\left|u_{0}\right\rangle\left\langle u_{0}\right|\right]}\right)$. Assume that the measured value $\left(q_{1}^{0}, p_{2}^{0}, p_{2}^{0}\right)\left(\in \mathbb{R}^{3}\right)$. That is,

$$
\begin{aligned}
& \left(q_{1}^{0}, p_{2}^{0}\right) \\
& \text { of } \left.A_{1} \text {,the momentum of } A_{2}\right)
\end{aligned} \underset{\text { the momentum of } A_{2}}{p_{2}^{0}}
$$

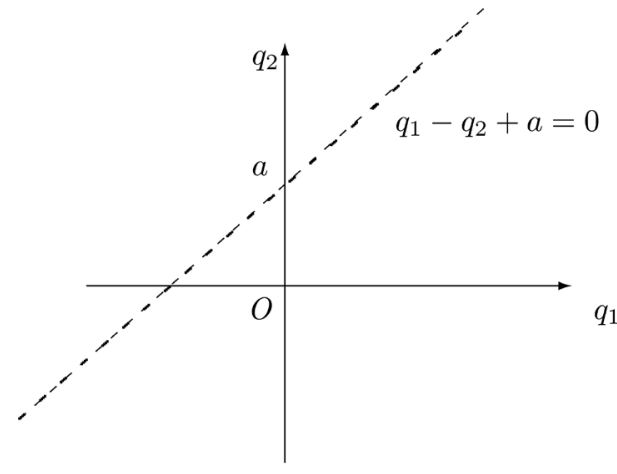

"the dashed line" $\approx$ "the support of $u_{0} "$

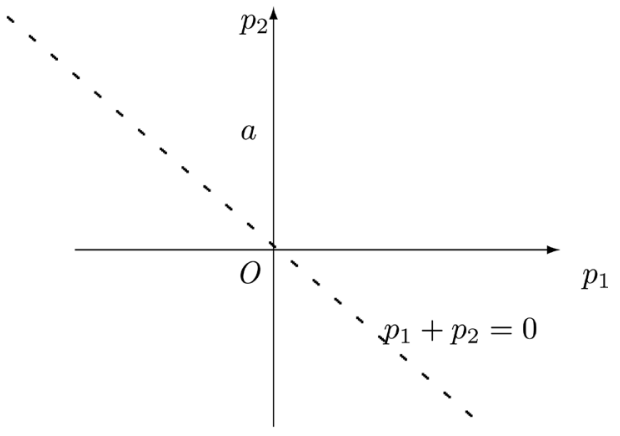

"the dashed line" $\approx$ "the support of $\widehat{u_{0}} "$

Figure 4. The supports of $u_{0}$ and $\widehat{u_{0}} \quad\left(0<\epsilon, \frac{1}{\sigma} \ll 1\right)$. 
$\left(\mathrm{Z}_{2}\right)$ Let $\mathrm{O}_{2}=\left(\mathbb{R}^{2}, \mathcal{B}_{\mathbb{R}^{2}}, F_{2}\right)$ in $B(H)\left(=B\left(L^{2}\left(\mathbb{R}^{2}\right)\right)\right)$ be the observable representation of $\left(I \otimes P_{2}\right) \times\left(P_{1} \otimes I\right)$. And consider the measurement $\mathrm{M}_{B(H)}\left(\mathrm{O}_{2}=\left(\mathbb{R}^{2}, \mathcal{B}_{\mathbb{R}^{2}}, F_{2}\right), S_{\left[\left|u_{0}\right\rangle\left\langle u_{0}\right|\right]}\right)$. Assume that the measured value $\left(p_{2}^{0},-p_{2}^{0}\right)\left(\in \mathbb{R}^{3}\right)$. That is,

$$
\underset{\text { the momentum of } A_{2}}{p_{2}^{0}} \Rightarrow \begin{gathered}
-p_{2}^{0} \\
\text { the momentum of } A_{1}
\end{gathered}
$$

$\left(Z_{3}\right)$ Therefore, if syllogism holds, we may conclude that

$$
\left(q_{1}^{0}, p_{2}^{0}\right) \Rightarrow \begin{gathered}
-p_{2}^{0} \\
\text { the momentum of } A_{1}
\end{gathered}
$$

(that is, the momentum of $A_{1}$ is equal to $-p_{2}^{0}$ ).

But, the above argument (particularly, syllogism [i.e., $\left(\mathrm{Z}_{1}\right)$ and $\left(\mathrm{Z}_{2}\right)$ imply $\left(Z_{3}\right)$ ]") is not true. That is because

$\left(\mathrm{Z}_{4}\right) \quad\left(Q_{1} \otimes P_{2}\right) \times\left(I \otimes P_{2}\right)$ and $\left(I \otimes P_{2}\right) \times\left(P_{1} \otimes I\right) \quad$ (therefore, $\mathrm{O}_{1}$ and $\left.\mathrm{O}_{2}\right)$ do not commute, and thus, the simultaneous observable does not exist.

Thus, we cannot test the $\left(Z_{3}\right)$ experimentally.

\section{Conclusions}

From the scientific point of view, Wittgenstein's picture theory is unintelligible and cannot be called a theory. However, it is true that this theory has fascinated a lot of ordinary philosophy enthusiasts. Therefore, if their intuition is correct, it is reasonable to think that his theory is an incomplete description of a part of the very great theory (which qualifies as the "language of science").

Statistics is a kind of language of science, and it is very useful. However, it has little power to solve theoretical and philosophical problems. Thus, I cannot expect that statistics are behind Wittgenstein's picture theory. On the other hand, quantum language may not be handy, but it is theoretical. And thus, quantum language has a great power to solve many unsolved theoretical problems (e.g., Brain in a vat, Peirce's abduction, Monty-Hall problem, etc.) as listed in Chap. 12 of ref. [21]. Therefore I expected quantum language in this paper.

First I discussed logic in quantum language in Sec. 3. And I concluded that a class of classical binary projective measurements has local structure. This result reminds us of Wittgenstein's TLP (i.e.,Tractatus Logico philosophicus), in which he should have discussed "logic in the language of science" without the definition of "the language of science".. From this perspective, in Sec. 4, I proposed my understanding of Wittgenstein's picture theory. That is, I concluded that the proposition that Wittgenstein studied in TPL has a similar logical nature to a binary projective measurement in classical QL. If I believe in Wittgenstein's saying "The limits of my language mean the limits of my world", I can say that Wittgenstein's picture theory is realized by classical QL.

It should be noted that QL has a great power to solve a lot of fundamental philosophical unsolved problems (e.g., Leibniz-Clake correspondence, the problem of qualia, Mctaggart's paradox, etc. cf. Chap. 12 in ref. [21]). Thus, I believe 
that QL is the unified theory of metaphysics and further, the language of science. Therefore, I think that our result in Sec. 3 is exactly what Wittgenstein wanted to write about in TLP. If so, Sec. 4 is not needed.

Also, it should be noted that QL (e.g., Sec. 3 in this paper) bridges between Descartes = Kant philosophy (i.e., epistemology) and analytic philosophy (e.g., Sec. 4 in this paper) in term of "measurement".

TLP ends with the following famous sentences:

- (6.54): My propositions are elucidatory in this way: he who understands me finally recognizes them as senseless, when he has climbed out through them, on them, over them. (He must so to speak throw away the ladder, after he has climbed up on it.) He must transcend these propositions, and then he will see the world aright.

- (7): What we cannot speak about we must pass over in silence.

I believe that Wittgenstein's language (i.e., the language that he supposed in TLP) will be replaced by classical QL. However, a lot of verification is needed to solidify my belief. Someone may propose another stronger theory in which Wittgenstein's picture theory can be fully understood. However, it should be noted that my purpose is to create a powerful language for science (like Newton, Einstein, Bayes, Fisher, etc.), not to understand Wittgenstein's theory.

I hope that many readers will examine my proposal from various aspects.

\section{Conflicts of Interest}

The author declares no conflicts of interest regarding the publication of this paper.

\section{References}

[1] Wittgenstein, L. (1921) Tractatus Logico Philosophicus. Routledge and Kegan Paul, Oxford.

[2] Conant, J. and Diamond, C. (2004) On Reading the Tractatus Resolutely: Reply To Meredith Williams and Peter Sullivan. In: Kölbel, M. and Weiss, B., Eds., The Lasting Significance of Wittgenstein's Philosophy, Routledge, Abingdon-on-Thames.

[3] Michael, K. (2001) The Purpose of Tractarian Nonsense. Noûs, 35, 39-73. https://doi.org/10.1111/0029-4624.00287

[4] Ishikawa, S. (1997) Fuzzy Inferences by Algebraic Method. Fuzzy Sets and Systems, 87, 181-200. https://doi.org/10.1016/S0165-0114(96)00035-8

[5] Ishikawa, S. (1997) A Quantum Mechanical Approach to Fuzzy Theory. Fuzzy Sets and Systems, 90, 277-306. https://doi.org/10.1016/S0165-0114(96)00114-5

[6] Ishikawa, S. (1998) Fuzzy Logic in Measurements. Fuzzy Sets and Systems, 100, 291-300. https://doi.org/10.1016/S0165-0114(97)00154-1

[7] Ishikawa, S. (2000) Statistics in Measurements. Fuzzy Sets and Systems, 116, 141-154. https://doi.org/10.1016/S0165-0114(98)00280-2

[8] Ishikawa, S. (2006) Mathematical Foundations of Measurement Theory. Keio University Press Inc., Tokyo, 335 p. http://www.keio-up.co.jp/kup/mfomt/

[9] Ishikawa, S. (2019) Linguistic Copenhagen Interpretation of Quantum Mechanics; 
Quantum Language, Research Report, Dept. Math. Keio University, Ver. 5, KSTS/ RR-19/003, 473 p.

http://www.math.keio.ac.jp/academic/research pdf/report/2019/19003.pdf

[10] Ishikawa, S. (2011) A New Interpretation of Quantum Mechanics. Journal of Quantum Information Science, 1, 35-42. http://dx.doi.org/10.4236/jqis.2011.12005

http://www.scirp.org/journal/PaperInformation.aspx?paperID=7610

[11] Ishikawa, S. (2015) Linguistic Interpretation of Quantum Mechanics; Projection Postulate. Journal of Quantum Information Science, 5, 150-155.

http://dx.doi.org/10.4236/iqis.2015.54017 http://www.scirp.org/Journal/PaperInformation.aspx?PaperID=62464

[12] Ishikawa, S. (2017) Bell's Inequality Should Be Reconsidered in Quantum Language. Journal of Quantum Information Science, 7, 140-154.

https://doi.org/10.4236/jqis.2017.74011

http://www.scirp.org/Journal/PaperInformation.aspx?PaperID=80813

[13] Ishikawa, S. (2012) Quantum Mechanics and the Philosophy of Language: Reconsideration of Traditional Philosophies. Journal of Quantum Information Science, 2, 2-9. http://dx.doi.org/10.4236/jqis.2012.21002 http://www.scirp.org/journal/PaperInformation.aspx?paperID=18194

[14] Ishikawa, S. (2017) A Final Solution to Mind-Body Problem by Quantum Language. Journal of Quantum Information Science, 7, 48-56.

https://doi.org/10.4236/jqis.2017.72005

http://www.scirp.org/Journal/PaperInformation.aspx?PaperID=76391

[15] Ishikawa, S. (2018) Leibniz-Clarke Correspondence, Brain in a Vat, Five-Minute Hypothesis, McTaggart's Paradox, etc. Are Clarified in Quantum Language. Open Journal of Philosophy, 8, 466-480. https://doi.org/10.4236/ojpp.2018.85032 https://www.scirp.org/Journal/PaperInformation.aspx?PaperID=87862

[16] Ishikawa, S. (2008) Dynamical Systems, Measurements, Quantitative Language and Zeno's Paradoxes. Far East Journal of Dynamical Systems, 10, 277-292. http://www.pphmj.com/abstract/3595.htm

[17] Ishikawa, S. (2012) A Measurement Theoretical Foundation of Statistics. Applied Mathematics, 3, 283-292. http://dx.doi.org/10.4236/am.2012.33044 http://www.scirp.org/journal/PaperInformation.aspx?paperID=18109

[18] Ishikawa, S. (2012) Monty Hall Problem and the Principle of Equal Probability in Measurement Theory. Applied Mathematics, 3, 788-794.

http://dx.doi.org/10.4236/am.2012.37117 http://www.scirp.org/journal/PaperInformation.aspx?PaperID=19884

[19] Ishikawa, S. (2012) Ergodic Hypothesis and Equilibrium Statistical Mechanics in the Quantum Mechanical World View. World Journal of Mechanics, 2, 125-130.

http://dx.doi.org/10.4236/wjm.2012.22014

https://www.scirp.org/journal/paperinformation.aspx?paperid=18861

[20] Ishikawa, S. (2019) Philosophy of Science for Scientists: The Probabilistic Interpretation of Science. Journal of Quantum Information Science, 9, 140-154. https://doi.org/10.4236/jqis.2019.93007 https://www.scirp.org/Journal/paperinformation.aspx?paperid=95447

[21] Ishikawa, S. (2020) History of Western Philosophy from the Quantum Theoretical Point of View. Research Report, Department of Mathematics, Keio University, Yokohama, $296 \mathrm{p}$.

http://www.math.keio.ac.jp/academic/research_pdf/report/2020/20001.pdf

[22] von Neumann, J. (1932) Mathematical Foundations of Quantum Mechanics. Sprin- 
ger Verlag, Berlin

[23] Sakai, S. (1971) $C^{\star}$-Algebras and $W^{\star}$-Algebras, Ergebnisse der Mathematik und ihrer Grenzgebiete (Band 60). Springer-Verlag, Berlin, Heidelberg, New York.

[24] Yosida, K. (1980) Functional Analysis. Sixth Edition, Springer-Verlag, Berlin, Heidelberg.

[25] Davies, E.B. (1976) Quantum Theory of Open Systems. Academic Press, Cambridge.

[26] Holevo, A.S. (1982) Probabilistic and Statistical Aspects of Quantum Theory. NorthHolland Publishing Company, Amsterdam.

[27] Howard, D. (2004) Who Invented the "Copenhagen Interpretation"? A Study in Mythology. Philosophy of Science, 71, 669-682. https://doi.org/10.1086/425941

[28] Halmos, P.R. (1974) Naive Set Theory. Springer-Verlag, New York. https://doi.org/10.1007/978-1-4757-1645-0

[29] Einstein, A., Podolosky, B. and Rosen, N. (1935) Can Quantum-Mechanical Description of Reality Be Considered Completely? Physical Review, 47, 777-780. https://doi.org/10.1103/PhysRev.47.777

[30] Ishikawa, S. (1991) Uncertainty Relation in Simultaneous Measurements for Arbitrary Observables. Reports on Mathematical Physics, 29, 257-273.

https://doi.org/10.1016/0034-4877(91)90046-P 